\title{
Comparación de la técnica de digestibilidad in vitro con la in situ de diez forrajes en bovinos rumino-fistulados en el piedemonte llanero del Meta
}

\section{Comparison of digestibility technical in vitro with in situ of ten fodder in cattle \\ "rumino-fistulados" in the "Piedemonte Llanero of Meta"}

\author{
${ }^{1}$ Navarro CA, ${ }^{1}$ Díaz JC, ${ }^{2}$ Roa ML y ${ }^{3}$ Cuellar E \\ ${ }^{1}$ Médicos Veterinarios Zootecnistas. ${ }^{2} Z$ ootecnista, MSc. Docente Universidad de los Llanos. \\ ${ }^{3}$ Médica Veterinaria y Zootecnista, Esp. Laboratorio de Nutrición Animal, UNILLANOS \\ cesar.navarro@unillanos.edu.co
}

Recibido 16 de Junio 2011, Aceptado 12 de Agosto 2011

\section{RESUMEN}

El consumo y la digestibilidad son temas de gran interés para los nutricionistas, puesto que en la producción animal se requiere de alimentos con alta aceptación y excelente aprovechamiento, con bajas pérdidas de nutriente por excretas. Atendiendo a lo anterior, esta investigación se realizó en UNILLANOS Villavicencio, consideró como objetivo principal evaluar dos técnicas de digestibilidad (in situ en rumen e in vitro) de 6 leguminosas, 2 gramíneas y 2 arbustivas no leguminosas a las que se les determinó la digestibilidad de la materia seca (MS), fibra detergente neutro (FDN) y nitrógeno total (NT). Se escogieron las leguminosas: Bauhinia variegata, Piptadenia (Anadenanthera) peregrina, Cratylia argentea, Brownea ariza, Gliricidia sepium y Delonix regia; las gramíneas Brachiaria decumbens y Pennisetum purpureum; y las arbustivas no leguminosas Tithonia diversifolia e Hibiscus rosasinensis. Las muestras fueron recolectadas de las parcelas de forrajes de la Universidad, a una edad de rebrote de 60 días. A los forrajes secos se les realizó un análisis nutricional preliminar, en el cual se determinó MS, NT y FDN. En las pruebas in situ en rumen se utilizó la técnica con bolsas de nylon, en cada una se incubaron $8 \mathrm{gr}$ de materia seca de cada forraje por tres repeticiones en cada hora $(6,12,24,48$ y 72$)$, en dos novillas criollas cruzadas de $380 \mathrm{~kg}$, fistuladas a nivel del rumen, las cuales estaban en pastoreo continuo con $B$. decumbens, sal mineralizada y agua a voluntad. Las pruebas in vitro fueron realizadas en el Laboratorio de Nutrición Animal, para ello se incubó $1 \mathrm{gr}$ de muestra con líquido ruminal durante 72 horas a $38^{\circ} \mathrm{C}$, y en el residuo obtenido se determinó la MS, FDN y NT. Con la 
información obtenida se estableció la curva y tasa de degradación de la MS, FDN y NT a las $6,12,24,48$ y 72 horas, de cada uno de los 10 forrajes. En las pruebas in situ se realizaron mediciones de $\mathrm{pH}$ y nitrógeno amoniacal $(\mathrm{N}-\mathrm{NH} 3)$ a las 0,6 y 12 horas en el líquido ruminal. El diseño estadístico fue un análisis de varianza para determinar los rangos de desviación entre las dos técnicas. Las variables evaluadas fueron los promedios in situ e in vitro de la degradación ruminal $(6,12,24,48$ y 72$)$ de la MS, FDN y NT de los 10 forrajes. Los datos se analizaron aplicando la prueba T-Student para comparación entre medias. La diferencia estadística $(\mathrm{P}<0.05)$ de los promedios in situ e in vitro de digestibilidad de la MS, fue del $100 \%$ en $B$. variegata, $C$. argentea, $B$. ariza, $H$. rosa-sinensis y $G$. sepium; $60 \%$ en $B$. decumbens, $P$. purpureum, $T$. diversifolia y Piptadenia peregrina; y en $D$. regia fue del $80 \%$. En la FDN fue del $100 \%$ en B. decumbens, $T$. diversifolia, C. argéntea, Piptadenia peregrina, $B$. ariza, $H$. rosa-sinensis y $G$. sepium; $80 \%$ en $P$. purpureum y $B$. variegata; y en $D$. regia fue del $60 \%$. En el NT fue del $100 \%$ en $C$. argéntea, B. ariza y G. sepium; $80 \%$ en $B$. decumbens, $P$. purpureum, B. variegata, Piptadenia peregrina y $D$. regia; y en $T$. diversifolia e $H$. rosasinensis fue del $60 \%$. Además, se encontró en el $63,333 \%$ de los casos una mejor tasa de degradación mediante la técnica de digestibilidad in situ. En conclusión, existen diferencias entre las técnicas de digestibilidad in situ e in vitro para estimar la digestibilidad de la MS, FDN y NT en los forrajes estudiados.

Palabras clave: Digestión, gramíneas, leguminosas, arbustos no leguminosos.

\section{ABSTRACT}

Consumption and digestibility are topics of great interest to nutritionists, as in animal foods requires high acceptance and excellent use, with low nutrient losses by excreta. Based on the foregoing, this research was conducted, at the Llanos University, Villavicencio, the main objective was to evaluate two digestibility techniques (in situ in rumen and in vitro) of six legumes, two grasses and two non-legumes shrub to which were determined digestibility of dry matter (DM), neutral detergent fiber (NDF) and total nitrogen (TN). Legumes were: Bauhinia variegata, Piptadenia (Anadenanthera) peregrina, Cratylia argentea, Brownea ariza, Gliricidia sepium and Delonix regia, the grasses: Brachiaria decumbens and Pennisetum purpureum, and non-leguminous shrub: Tithonia diversifolia and Hibiscus rosa-sinensis. The Samples were collected from of fodder of the University, the fodders were utilized 60 days 
after of regrowth. The dry fodders underwent a preliminary nutritional analysis, which were determined DM, NDF and TN. In the in situ tests rumen technique was used with nylon bags, each incubated 8 grams of DM feed every three replicates at each hour $(6,12,24,48$ y 72$)$, in two creole heifers cross fistulated at the rumen, which were in continuous grazing Brachiaria decumbens, mineralized salt and water ad libitum. In vitro tests were conducted at the Laboratory of Animal Nutrition, for this $1 \mathrm{~g}$ of the sample was incubated in rumen fluid for 72 hours at $38^{\circ} \mathrm{C}$, and in the residue obtained were determined DM, NDF and TN. With the information was established the curve and rate of degradation of DM, NDF and NT at 6, 12, 24, 48 and 72 hours in ten forages. In the in situ test was evaluated the $\mathrm{pH}$ and the ammonia nitrogen (N-NH3) at 0,6 and 12 hours in the rumen fluid. The design was an analysis of variance to determine the range of deviation between the two techniques. The variables evaluated were the average in situ and in vitro digestibility in ruminal fluid $(6,12,24,48$ and 72) of DM, NDF and TN of the 10 forages. The results were analyzed using T-Student test for comparison between two techniques. The statistical difference $(\mathrm{P}<0.05)$ from the average in situ and in vitro digestibility of DM was $100 \%$ in: B. variegata, C. argentea, B. ariza, H. rosasinensis and $G$. sepium, $60 \%$ in B. decumbens, P. purpureum, Piptadenia peregrina, $T$. diversifolia; and in D. regia was $80 \%$. The NDF was $100 \%$ in: B. decumbens, T. diversifolia, C. argentea, Piptadenia peregrina, B. ariza, $H$. rosa-sinensis and G. sepium; $80 \%$ in $P$. purpureum and $B$. variegata; and $D$. regia was $60 \%$. In the TN was $100 \%$ in: $C$ argentea, $B$. ariza and G. sepium; $80 \%$ in B. decumbens, P. purpureum, B. variegata, Piptadenia peregrina and $D$. regia; and in $T$. diversifolia and $H$. rosa-sinensis was $60 \%$. It was also found in the $63.333 \%$ of cases a better rate of degradation by the technique of in situ digestibility. In conclusion there are statistical differences $(P<0.05)$ between the techniques of digestibility in situ and in vitro to estimate the dry matter digestibility, neutral detergent fiber and total nitrogen in forages studied.

Keywords: Digestion, grasses, legumes, non-legumes shrub.

\section{INTRODUCCIÓN}

La digestibilidad hace referencia a la cantidad de alimento que desaparece en el tracto digestivo o en un procedimiento de laboratorio debido a su solubilización o ataque por los microorganismos anaerobios ruminales (Giraldo et al., 2006). Mientras que, la degradabilidad 
hace referencia a la cantidad de alimento que se descompone en sus elementos integrantes, mediante procesos biológicos o químicos. A diferencia de la degradabilidad, la digestibilidad de los forrajes permite estimar la proporción de nutrientes presentes en el alimento, que tienen potencial de ser absorbidos por el tracto digestivo (Giraldo et al., 2006). La degradabilidad ruminal, tiene un valor relativo, pues depende de dos aspectos: velocidad de degradación y velocidad de transito ruminal. A su vez, la primera se determina por la solubilidad y estructura molecular, y actividad de los microorganismos y puede ser afectada por: el pH, el tamaño de partícula, la relación forraje: concentrado, y otros factores como la ingestión de agua o materia seca, alterando la degradabilidad ruminal (Rosero y Posada, 2007). La energía es limitante en todo sistema de alimentación, de allí la importancia de su valoración en los alimentos (Torres et al., 2009). El valor energético se establece mediante: ensayos de digestibilidad directa (Sosa et al., 2006) o de forma indirecta estimando digestibilidades con técnicas in situ e in vitro, ó empleando enzimas celulolíticas (Arce et al., 2003). La técnica in situ utiliza bolsas sintéticas para medir la digestión de los forrajes a nivel ruminal, consiste en colocar la muestra en la bolsa e incubarla en rumen de animales fistulados. Esta técnica permite determinar simultáneamente la cantidad de la muestra ingerida y la tasa a la cual la digestión se realiza. Se utiliza principalmente cuando se requiere observar el efecto de las condiciones ruminales sobre la digestión de un número limitado de muestras. La utilidad y confiabilidad de esta técnica depende de factores tales como la cantidad de la muestra, del tamaño de la bolsa y de la partícula de la muestra (Torres et al., 2009). Por otro lado, los métodos in vitro que han sido utilizados ampliamente desde su introducción son el de Tilley y Terry (1980) y el de Van Soest et al. (1991), considerados los procedimientos más exactos para la predicción de la digestibilidad en rumiantes. El método de Tilley y Terry se considera de referencia para calcular la digestibilidad en rumiantes, ha sido modificado y adaptado según el tipo de alimento, al igual que se han probado diferentes tampones de dilución para ajustar el pH del inóculo. Pese a su exactitud y modificaciones, este método requiere de mucho tiempo y trabajo, además cada alimento debe incubarse por separado, limitando el número de muestras a ser analizadas (Giraldo et al., 2006). Así mismo, la técnica desarrollada por Van Soest et al., (1991), supone una alternativa al método de Tilley y Terry (1980), ya que permite una valoración más rápida de los alimentos sin afectar negativamente la precisión del valor obtenido. Este procedimiento consiste en una incubación de los alimentos con líquido ruminal durante 48 
horas a $38^{\circ} \mathrm{C}$, seguido del tratamiento del residuo obtenido con una solución neutro detergente durante 1 hora a $100^{\circ} \mathrm{C}$, y los valores obtenidos se consideran una estimación de la digestibilidad real de los alimentos (Bochi-Brum, et al., 1999). El inconveniente de la técnica in vitro reside en la variabilidad de sus resultados, debido a que la microflora ruminal está influenciada por el tipo y cantidad de dieta proporcionada al animal (Torres et al., 2009).

\section{MATERIALES Y MÉTODOS}

Esta investigación se realizó en el municipio de Villavicencio, en la granja de UNILLANOS sede Barcelona y en el Laboratorio de Nutrición Animal ubicados en el kilómetro 12 vía Puerto López, con una altitud de 465 metros sobre el nivel del mar, temperatura promedio de $27^{\circ} \mathrm{C}$ y precipitación anual entre 1.900 y 3.250 milímetros (Roa et al., 2010). Para las pruebas in situ se utilizaron dos novillas de $380 \mathrm{~kg}$ criollas cruzadas, fistuladas en rumen, las cuales estaban en pastoreo continuo con Brachiaria decumbens, sal mineralizada y agua a voluntad.

Las muestras fueron recolectadas de las parcelas de forrajes de la Universidad, a una edad de rebrote de 60 días. Se deshidrataron en una estufa de aire forzado a $72^{\circ} \mathrm{C}$ por 72 horas, posteriormente se molieron (criba de $1 \mathrm{~mm}$ ) para homogenizar el tamaño de la partícula. A los forrajes secos pasto amargo (B. decumbens), kingrass ( $P$.purpureum), botón de oro ( $T$. diversifolia), casco de vaca (B. variegata), yopo ( $A$. (Piptadenia) peregrina, veranera ( $C$. argentea), palo de cruz (B. ariza), cayeno ( $H$. rosa-sinensis), matarratón ( $G$. sepium) y acacia roja (D. regia), se les realizó un análisis preliminar, determinando materia seca (MS), nitrógeno total (NT) y fibra detergente neutro (FDN), tres repeticiones por forraje, siguiendo la metodología de la AOAC, 2006.

En las pruebas in situ en rumen se utilizó la técnica con bolsas de nylon (Mertens, 2002), cuyo tamaño fue de $20 \times 10 \mathrm{~cm}$ con un poro promedio de 50 micras, para evitar la salida de forraje. Estas bolsas se secaron previamente a $60^{\circ} \mathrm{C}$ por 24 horas para llevarlas a peso constante y en cada una se colocaron $8 \mathrm{gr}$ de materia seca de cada arbusto o forraje por tres repeticiones en cada hora $(6,12,24,48$ y 72$)$, es decir, se incubaron 15 bolsas por forraje para un total de 150, las cuales fueron colocadas en la porción ventral del rumen, dejándoles cierta libertad de movimiento, y así quedaran expuestas a las condiciones del rumen. Para sostenerlas, fueron amarradas a una cadena conservando una distancia entre la cánula y las primeras bolsas de $50 \mathrm{~cm}$ (Fotografía 1), Colocándolas en forma secuencial para ser 
extraídas (Fotografías 2. y 3.) de acuerdo a la hora correspondiente de degradación, se realizó su lavado hasta retirar los residuos del rumen, (Fotografía 4.). Estas pruebas fueron divididas en dos etapas: en la primera se inocularon 45 bolsas en cada animal, y en la segunda etapa las restantes 30 bolsas en cada animal. Además, en las pruebas in situ se realizaron mediciones de $\mathrm{pH}$ (Fotografía 6.) y nitrógeno amoniacal (N-NH3) (Fotografía 10) en el líquido ruminal a las 0,6 y 12 horas. Los 5 forrajes de mayor contenido proteico, botón de oro, veranera, matarratón, cayeno y acacia roja se incubaron en el primer animal y los 5 forrajes restantes de menor contenido proteico, pasto amargo, kingrass, casco de vaca, yopo, y palo de cruz en el segundo animal.

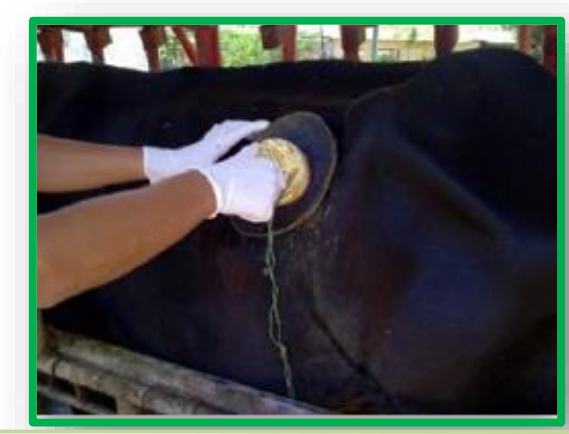

Fotografía 1. Retiro tapa de cánula

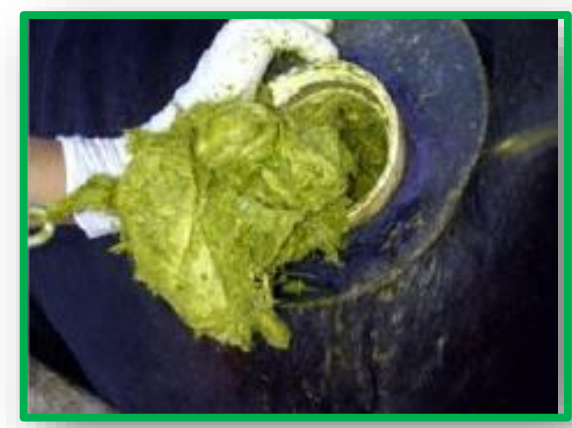

Fotografía 3. Extracción de bolsas

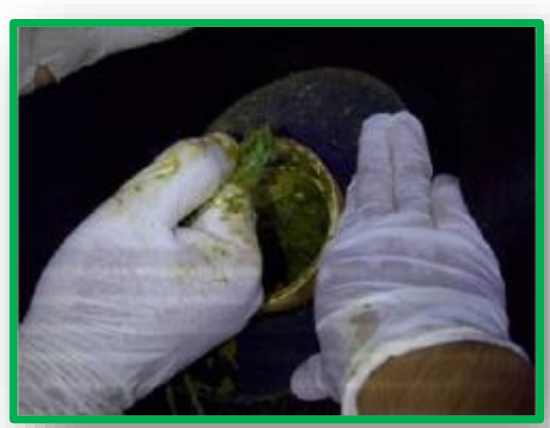

Fotografía 2. Extracción de cadenas

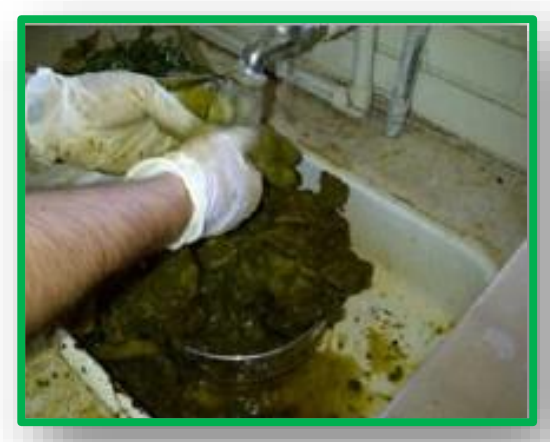

Fotografía 4. Lavado de bolsas

Las pruebas in vitro fueron realizadas en el Laboratorio de Nutrición Animal, AOAC (2006), para ello se incubaron los forrajes con líquido ruminal durante 72 horas a $38^{\circ} \mathrm{C}$, seguido del tratamiento del residuo obtenido (Fotografía 7) con una solución neutro-detergente durante 1 hora a $100^{\circ} \mathrm{C}$ (Fotografía 8). La extracción del líquido se realizó manualmente y en horas de la mañana (7 a.m.) quitando la tapa de la cánula de los dos animales rumino-fistulados, se retiró toda la ingesta situada en la parte alta del rumen y se extrajo la parte semilíquida que 
fue filtrada en un vaso estéril de $250 \mathrm{ml}$, utilizando una gasa. El material extraído fue mezclado con la solución de Mc Dougall previo al inicio de la incubación. El tubo con inoculo fue mantenido a una temperatura de $38^{\circ} \mathrm{C}$, en un baño de maría (Fotografía 9) y lleno a capacidad evitando la oxigenación del material. La relación buffer: líquido ruminal fue de 3: 1, es decir que se utilizó $3.75 \mathrm{ml}$ de buffer por cada $1.25 \mathrm{ml}$ de liquido ruminal para las 72 horas de incubación. La inoculación de cada forraje, se hizo en tubos de $10 \mathrm{ml}$ procesando 3 muestras para cada hora $(6,12,24,48$ y 72), utilizando 150 tubos. El contenido de los tubos fue agitado suavemente una hora después de iniciada la incubación, seguido por dos agitaciones adicionales en el primer día, y en el segundo día tres veces. Se utilizó un volumen de $5 \mathrm{ml}$ de inoculo para $1 \mathrm{gr}$ de muestra (materia seca de cada forraje).

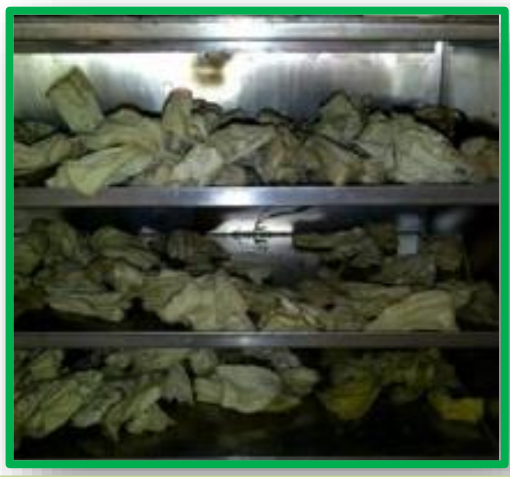

Fotografía 5. Secado de bolsas

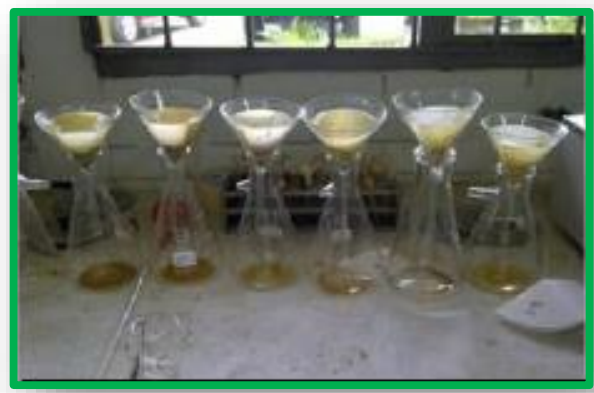

Fotografía 7. Obtención de residuo

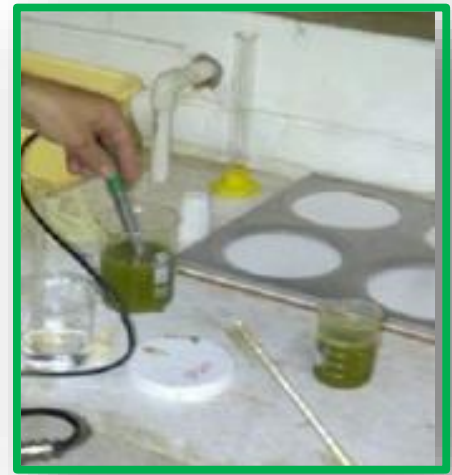

Fotografía 6. Determinación pH

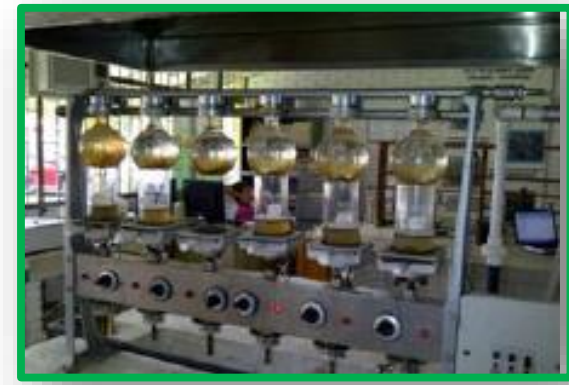

Fotografía 8. Digestión equipo de Fibra

Con la información obtenida se estableció la curva y tasa de degradación de la MS, FDN y NT a las 6, 12, 24, 48 y 72 horas, de cada uno de los 10 forrajes, para ello los datos experimentales fueron ajustados mediante la fórmula de McDonall y la función solver del Software Excel, Microsoft $\AA$, ya que hay reportes de que su uso permite una estimación 
confiable y rápida. (Fernández, 2004). El diseño estadístico fue un análisis de varianza para determinar los rangos de desviación entre las dos técnicas, para establecer la tendencia de los datos. Las variables evaluadas fueron los promedios de la degradación ruminal $(6,12,24$, 48 y 72 horas) de la materia seca, fibra detergente neutro y proteína cruda de los 10 forrajes. Los datos se analizaron aplicando la prueba T-Student para comparación entre medias.

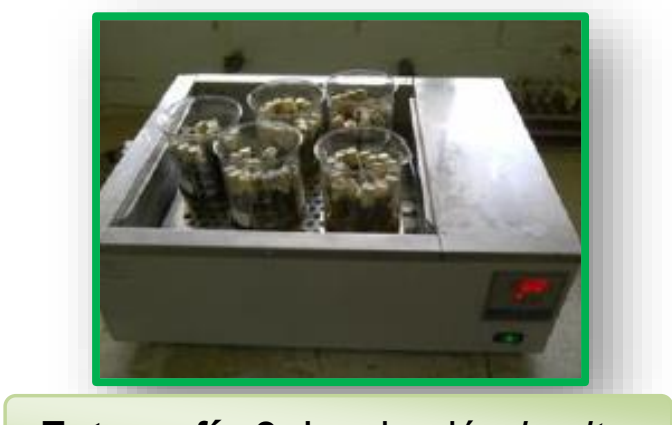

Fotografía 9. Incubación in vitro

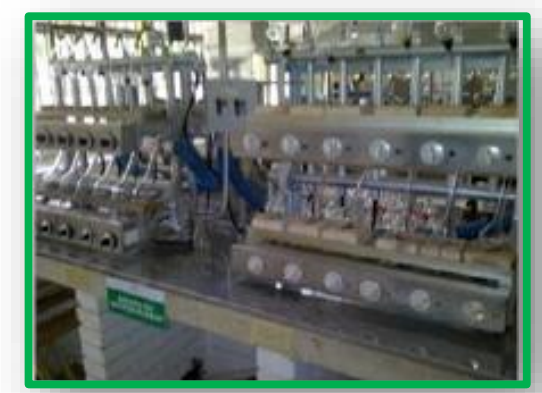

Fotografía 10. Determinación nitrógeno total y amoniacal.

\section{RESULTADOS}

En las gramíneas las digestibilidades in vitro de la MS, FDN y NT del pasto amargo fueron mayores $(P<0,05)$ que las in situ, excepto en: MS a las 48 y 72 horas, y NT a las 24 horas, en estos casos no hubo diferencia (Tabla 1 y Figura 1).

Tabla 1. Digestibilidad in situ Vs in vitro pasto amargo (Brachiaria decumbens)

\begin{tabular}{|c|c|c|c|c|c|c|}
\hline \multirow{2}{*}{ Horas } & \multicolumn{2}{|c|}{ Digestibilidad MS (\%) * } & \multicolumn{2}{c|}{ Digestibilidad FDN (\%) ** } & \multicolumn{2}{c|}{ Digestibilidad Proteina (\%) } \\
\cline { 2 - 7 } & In Situ & In Vitro & In Situ & In Vitro & In Situ & In vitro \\
\hline 6 & $17,300^{\mathrm{a}}$ & $28,460^{\mathrm{b}}$ & $43,436^{\mathrm{a}}$ & $51,928^{\mathrm{b}}$ & $30,324^{\mathrm{a}}$ & $39,842^{\mathrm{b}}$ \\
\hline 12 & $19,942^{\mathrm{a}}$ & $39,703^{\mathrm{b}}$ & $45,989^{\mathrm{a}}$ & $53,492^{\mathrm{b}}$ & $42,005^{\mathrm{a}}$ & $51,278^{\mathrm{b}}$ \\
\hline 24 & $25,938^{\mathrm{a}}$ & $44,990^{\mathrm{b}}$ & $46,894^{\mathrm{a}}$ & $54,213^{\mathrm{b}}$ & $52,232^{\mathrm{a}}$ & $57,050^{\mathrm{a}}$ \\
\hline 48 & $45,250^{\mathrm{a}}$ & $48,202^{\mathrm{a}}$ & $48,124^{\mathrm{a}}$ & $55,091^{\mathrm{b}}$ & $62,375^{\mathrm{a}}$ & $60,311^{\mathrm{b}}$ \\
\hline 72 & $48,805^{\mathrm{a}}$ & $50,312^{\mathrm{a}}$ & $49,566^{\mathrm{a}}$ & $57,900^{\mathrm{b}}$ & $70,082^{\mathrm{a}}$ & $76,546^{\mathrm{b}}$ \\
\hline
\end{tabular}

* Materia Seca. ** Fibra Detergente Neutro. Letras distintas en las columnas de cada nutriente in situ e in vitro son diferentes $(\mathrm{P}<0,05)$.

La cinética de la degradación de la MS fue similar en las dos técnicas de digestibilidad, mientras que la degradabilidad efectiva ruminal (DER) de la FDN y NT fue mayor $(\mathrm{P}<0.05)$ mediante la estimación in vitro (Tablas 11, 12 y 13). Con el kingrass las digestibilidades in vitro de la MS, FDN y NT fueron mayores $(P<0,05)$ que las in situ, excepto en: MS a las 48 y 72 horas, FDN a las 12 horas y NT a las horas, Tabla 2 y Figura 2). La cinética de la 
degradación de la MS y NT fue similar en las dos técnicas de digestibilidad, mientras que en la DER de la FDN se hallo un valor más alto $(P<0,05)$ mediante la técnica in vitro (Tablas 11 , 12 y 13).

Tabla 2. Digestibilidad in situ Vs in vitro kingrass (Pennisetum purpureum)

\begin{tabular}{|c|c|c|c|c|c|c|}
\hline \multirow{2}{*}{ Horas } & \multicolumn{2}{|c|}{ Digestibilidad MS (\%) * } & \multicolumn{2}{c|}{ Digestibilidad FDN (\%) ** } & \multicolumn{2}{c|}{ Digestibilidad Proteina (\%) } \\
\cline { 2 - 7 } & In Situ & In Vitro & In Situ & In Vitro & In Situ & In vitro \\
\hline 6 & $11,727^{\mathrm{a}}$ & $20,881^{\mathrm{b}}$ & $28,479^{\mathrm{a}}$ & $32,155^{\mathrm{b}}$ & $24,476^{\mathrm{a}}$ & $40,732^{\mathrm{b}}$ \\
\hline 12 & $12,851^{\mathrm{a}}$ & $27,963^{\mathrm{b}}$ & $30,650^{\mathrm{a}}$ & $32,738^{\mathrm{a}}$ & $32,951^{\mathrm{a}}$ & $44,638^{\mathrm{b}}$ \\
\hline 24 & $24,563^{\mathrm{a}}$ & $31,222^{\mathrm{b}}$ & $32,325^{\mathrm{a}}$ & $33,333^{\mathrm{b}}$ & $39,491^{\mathrm{a}}$ & $47,447^{\mathrm{b}}$ \\
\hline 48 & $33,173^{\mathrm{a}}$ & $36,774^{\mathrm{a}}$ & $34,246^{\mathrm{a}}$ & $35,780^{\mathrm{b}}$ & $45,235^{\mathrm{a}}$ & $50,248^{\mathrm{b}}$ \\
\hline 72 & $36,584^{\mathrm{a}}$ & $44,247^{\mathrm{a}}$ & $35,791^{\mathrm{a}}$ & $38,425^{\mathrm{b}}$ & $55,738^{\mathrm{a}}$ & $56,211^{\mathrm{a}}$ \\
\hline
\end{tabular}

* Materia Seca. ${ }^{* *}$ Fibra Detergente Neutro. Letras distintas en las columnas de cada nutriente in situ e in vitro son diferentes $(\mathrm{P}<0,05)$.

En las no leguminosas las digestibilidades in situ de la MS, FDN y NT del botón de oro fueron mayores $(P<0,05)$ que las in vitro, excepto en: MS a las 24 horas, y a las 6 y 48 horas fueron similares; y NT a las 12 horas, y a las 6 y 24 horas no hubo diferencia (Tabla 3 - Figura 3).

Tabla 3. Digestibilidad in situ Vs in vitro botón de oro (Tithonia diversifolia)

\begin{tabular}{|c|c|c|c|c|c|c|}
\hline \multirow{2}{*}{ Horas } & \multicolumn{2}{|c|}{ Digestibilidad MS (\%) * } & \multicolumn{2}{|c|}{ Digestibilidad FDN (\%) ** } & \multicolumn{2}{c|}{ Digestibilidad Proteina (\%) } \\
\cline { 2 - 7 } & In Situ & In Vitro & In Situ & In Vitro & In Situ & In vitro \\
\hline 6 & $19,714^{\mathrm{a}}$ & $24,346^{\mathrm{a}}$ & $39,957^{\mathrm{b}}$ & $17,435^{\mathrm{a}}$ & $34,483^{\mathrm{a}}$ & $37,537^{\mathrm{a}}$ \\
\hline 12 & $32,966^{\mathrm{b}}$ & $29,009^{\mathrm{a}}$ & $40,987^{\mathrm{b}}$ & $20,641^{\mathrm{a}}$ & $42,459^{\mathrm{a}}$ & $47,185^{\mathrm{b}}$ \\
\hline 24 & $37,044^{\mathrm{a}}$ & $41,793^{\mathrm{b}}$ & $41,562^{\mathrm{b}}$ & $21,748^{\mathrm{a}}$ & $51,071^{\mathrm{a}}$ & $51,844^{\mathrm{a}}$ \\
\hline 48 & $48,816^{\mathrm{a}}$ & $43,703^{\mathrm{a}}$ & $42,337^{\mathrm{b}}$ & $24,282^{\mathrm{a}}$ & $62,030^{\mathrm{b}}$ & $53,813^{\mathrm{a}}$ \\
\hline 72 & $65,819^{\mathrm{b}}$ & $45,366^{\mathrm{a}}$ & $44,318^{\mathrm{b}}$ & $28,603^{\mathrm{a}}$ & $67,021^{\mathrm{b}}$ & $57,511^{\mathrm{a}}$ \\
\hline
\end{tabular}

* Materia Seca. ${ }^{* *}$ Fibra Detergente Neutro. Letras distintas en las columnas de cada nutriente in situ e in vitro son diferentes $(\mathrm{P}<0,05)$.

La cinética de la degradación fue diferente en todos los nutrientes, la DER de la MS, FDN y NT estimada mediante la técnica in situ fue mayor $(P<0,05)$ (Tablas 11, 12 y 13). Así mismo, las digestibilidades in situ de la MS y NT del cayeno fueron mayores $(P<0,05)$ que las in vitro, excepto en: MS a las 6 y 72 horas, y NT a las 6 y 12 horas fueron similares; siendo menores en la FDN (Tabla 4 - Figura 8). Al igual que en el caso anterior, la cinética de la degradación fue diferente en todos los nutrientes, debido a que la DER de la MS y NT fue mayor $(P<0,05)$ en la estimación mediante la técnica in situ, mientras que en la FDN fue menor. (Tablas 11, 12 y 13). 
En las leguminosas las digestibilidades in vitro de la MS y NT de la veranera fueron mayores $(\mathrm{P}<0,05)$ que las in situ, y en la FDN fueron menores (Tabla 5 - Figura 5). La cinética de la degradación fue diferente en todos los nutrientes, puesto que la DER de la MS y FDN estimada mediante la técnica in situ fue mayor $(\mathrm{P}<0,05)$, mientras que la del NT fue menor (Tablas 11,12 y 13 ).

Tabla 4. Digestibilidad in situ Vs in vitro cayeno (Hibiscus rosa-sinensis)

\begin{tabular}{|c|c|c|c|c|c|c|}
\hline \multirow{2}{*}{ Horas } & \multicolumn{2}{|c|}{ Digestibilidad M.S. (\%) } & \multicolumn{2}{|c|}{ Digestibilidad FDN (\%) } & \multicolumn{2}{c|}{ Digestibilidad Proteina (\%) } \\
\cline { 2 - 7 } & In Situ & In Vitro & In Situ & In Vitro & In Situ & In vitro \\
\hline 6 & $21,067^{\mathrm{a}}$ & $27,047^{\mathrm{b}}$ & $45,402^{\mathrm{a}}$ & $48,162^{\mathrm{b}}$ & $31,290^{\mathrm{a}}$ & $37,014^{\mathrm{a}}$ \\
\hline 12 & $28,154^{\mathrm{b}}$ & $35,601^{\mathrm{a}}$ & $46,283^{\mathrm{a}}$ & $49,252^{\mathrm{b}}$ & $43,245^{\mathrm{a}}$ & $41,013^{\mathrm{a}}$ \\
\hline 24 & $51,230^{\mathrm{b}}$ & $42,278^{\mathrm{a}}$ & $47,339^{\mathrm{a}}$ & $51,078^{\mathrm{b}}$ & $54,703^{\mathrm{b}}$ & $46,675^{\mathrm{a}}$ \\
\hline 48 & $72,212^{\mathrm{b}}$ & $49,951^{\mathrm{a}}$ & $48,576^{\mathrm{a}}$ & $52,250^{\mathrm{b}}$ & $75,300^{\mathrm{b}}$ & $52,353^{\mathrm{a}}$ \\
\hline 72 & $83,178^{\mathrm{b}}$ & $58,741^{\mathrm{a}}$ & $48,856^{\mathrm{a}}$ & $53,325^{\mathrm{b}}$ & $85,179^{\mathrm{b}}$ & $62,002^{\mathrm{a}}$ \\
\hline
\end{tabular}

* Materia Seca. ${ }^{* *}$ Fibra Detergente Neutro. Letras distintas en las columnas de cada nutriente in situ e in vitro son diferentes $(\mathrm{P}<0,05)$.

Tabla 5. Digestibilidad in situ Vs in vitro veranera (Cratylia argentea)

\begin{tabular}{|c|c|c|c|c|c|c|}
\hline \multirow{2}{*}{ Horas } & \multicolumn{2}{|c|}{ Digestibilidad M.S. (\%) } & \multicolumn{2}{c|}{ Digestibilidad FDN (\%) } & \multicolumn{2}{c|}{ Digestibilidad Proteina (\%) } \\
\cline { 2 - 7 } & In Situ & In Vitro & In Situ & In Vitro & In Situ & In vitro \\
\hline 6 & $47,808^{\mathrm{a}}$ & $75,837^{\mathrm{b}}$ & $66,570^{\mathrm{b}}$ & $63,177^{\mathrm{a}}$ & $29,209^{\mathrm{a}}$ & $79,174^{\mathrm{b}}$ \\
\hline 12 & $59,438^{\mathrm{a}}$ & $79,870^{\mathrm{b}}$ & $66,976^{\mathrm{b}}$ & $65,487^{\mathrm{a}}$ & $35,142^{\mathrm{a}}$ & $82,650^{\mathrm{b}}$ \\
\hline 24 & $63,075^{\mathrm{a}}$ & $82,502^{\mathrm{b}}$ & $67,170^{\mathrm{b}}$ & $66,082^{\mathrm{a}}$ & $37,312^{\mathrm{a}}$ & $84,919^{\mathrm{b}}$ \\
\hline 48 & $71,079^{\mathrm{a}}$ & $84,428^{\mathrm{b}}$ & $67,386^{\mathrm{b}}$ & $66,412^{\mathrm{a}}$ & $40,680^{\mathrm{a}}$ & $86,579^{\mathrm{b}}$ \\
\hline 72 & $77,253^{\mathrm{a}}$ & $87,444^{\mathrm{b}}$ & $67,594^{\mathrm{b}}$ & $67,055^{\mathrm{a}}$ & $47,378^{\mathrm{a}}$ & $89,178^{\mathrm{b}}$ \\
\hline
\end{tabular}

${ }^{*}$ Materia Seca. ${ }^{*}$ Fibra Detergente Neutro. Letras distintas en las columnas de cada nutriente in situ e in vitro son diferentes $(\mathrm{P}<0,05)$.

De la misma forma, las digestibilidades in vitro de la MS y FDN de la acacia roja fueron mayores $(P<0,05)$ que las in situ, excepto en: MS a las 6 y 12 horas, y a las 72 fueron similares; lo mismo que en la FDN a las 6 y 12 horas el comportamiento fue similar; siendo menores en el NT $(P<0,05)$, excepto a las 6 horas (Tabla 6 - Figura 10). La cinética de la degradación de la MS fue similar en las dos técnicas de digestibilidad, mientras que la DER de la FDN presentó un valor más alto $(P<0,05)$ mediante la técnica in vitro, y uno más bajo en el NT (Tablas 11, 12 y 13).

Por el contrario, en el casco de vaca las digestibilidades in situ de la MS y FDN fueron mayores $(P<0,05)$ que las in vitro, excepto en: FDN a las 6 horas en la cual no hubo diferencia, mientras que para el NT fueron menores $(P<0,05)$, excepto a las 72 horas, en donde el comportamiento fue similar (Tabla 7 - Figura 4). La cinética de la degradación del 
NT fue similar en las dos técnicas de digestibilidad, mientras que la DER de la MS y FDN estimada mediante la técnica in situ fue mayor $(\mathrm{P}<0,05)$ (Tablas 11,12 y 13).

Tabla 6. Digestibilidad in situ Vs in vitro acacia roja (Delonix regia)

\begin{tabular}{|c|c|c|c|c|c|c|}
\hline \multirow{2}{*}{ Horas } & \multicolumn{2}{|c|}{ Digestibilidad M.S. (\%) } & \multicolumn{2}{|c|}{ Digestibilidad FDN (\%) } & \multicolumn{2}{c|}{ Digestibilidad Proteina (\%) } \\
\cline { 2 - 7 } & In Situ & In Vitro & In Situ & In Vitro & In Situ & In vitro \\
\hline 6 & $24,393^{\mathrm{a}}$ & $40,429^{\mathrm{b}}$ & $30,848^{\mathrm{a}}$ & $31,009^{\mathrm{a}}$ & $26,333^{\mathrm{a}}$ & $26,281^{\mathrm{a}}$ \\
\hline 12 & $27,524^{\mathrm{a}}$ & $41,173^{\mathrm{b}}$ & $32,228^{\mathrm{a}}$ & $32,880^{\mathrm{a}}$ & $30,746^{\mathrm{b}}$ & $27,446^{\mathrm{a}}$ \\
\hline 24 & $31,771^{\mathrm{a}}$ & $42,020^{\mathrm{b}}$ & $33,399^{\mathrm{a}}$ & $34,177^{\mathrm{b}}$ & $34,864^{\mathrm{b}}$ & $29,829^{\mathrm{a}}$ \\
\hline 48 & $38,179^{\mathrm{a}}$ & $43,116^{\mathrm{b}}$ & $34,341^{\mathrm{a}}$ & $35,596^{\mathrm{b}}$ & $40,774^{\mathrm{b}}$ & $34,001^{\mathrm{a}}$ \\
\hline 72 & $42,785^{\mathrm{a}}$ & $44,858^{\mathrm{a}}$ & $36,195^{\mathrm{a}}$ & $38,285^{\mathrm{b}}$ & $48,785^{\mathrm{b}}$ & $37,635^{\mathrm{a}}$ \\
\hline
\end{tabular}

* Materia Seca. ${ }^{* *}$ Fibra Detergente Neutro. Letras distintas en las columnas de cada nutriente in situ e in vitro son diferentes $(\mathrm{P}<0,05)$.

Tabla 7. Digestibilidad in situ Vs in vitro casco de vaca (Bauhinia variegata)

\begin{tabular}{|c|c|c|c|c|c|c|}
\hline \multirow{2}{*}{ Horas } & \multicolumn{2}{|c|}{ Digestibilidad M.S. (\%) } & \multicolumn{2}{|c|}{ Digestibilidad FDN (\%) } & \multicolumn{2}{c|}{ Digestibilidad Proteina (\%) } \\
\cline { 2 - 7 } & In Situ & In Vitro & In Situ & In Vitro & In Situ & In vitro \\
\hline 6 & $24,609^{\mathrm{b}}$ & $11,195^{\mathrm{a}}$ & $24,502^{\mathrm{a}}$ & $23,413^{\mathrm{a}}$ & $22,549^{\mathrm{a}}$ & $25,228^{\mathrm{b}}$ \\
\hline 12 & $28,787^{\mathrm{b}}$ & $23,599^{\mathrm{a}}$ & $30,245^{\mathrm{b}}$ & $25,405^{\mathrm{a}}$ & $27,257^{\mathrm{a}}$ & $31,384^{\mathrm{b}}$ \\
\hline 24 & $30,212^{\mathrm{b}}$ & $25,142^{\mathrm{a}}$ & $31,459^{\mathrm{b}}$ & $26,572^{\mathrm{a}}$ & $31,718^{\mathrm{a}}$ & $34,289^{\mathrm{b}}$ \\
\hline 48 & $31,327^{\mathrm{b}}$ & $27,066^{\mathrm{a}}$ & $35,865^{\mathrm{b}}$ & $27,430^{\mathrm{a}}$ & $35,577^{\mathrm{a}}$ & $38,508^{\mathrm{b}}$ \\
\hline 72 & $34,120^{\mathrm{b}}$ & $30,401^{\mathrm{a}}$ & $41,620^{\mathrm{b}}$ & $33,383^{\mathrm{a}}$ & $43,852^{\mathrm{a}}$ & $42,135^{\mathrm{a}}$ \\
\hline
\end{tabular}

* Materia Seca. ${ }^{* *}$ Fibra Detergente Neutro. Letras distintas en las columnas de cada nutriente in situ e in vitro son diferentes $(P<0,05)$.

Así mismo, en el yopo las digestibilidades in situ de la MS, FDN y NT fueron mayores $(P<0,05)$ que las in vitro, excepto en: MS a las 12 y 24 horas; y a las 6 y 72 horas no hubo diferencia, así como en el NT a las 72 horas (Tabla 8 - Figura 6). La cinética de la degradación de la MS y NT fueron similares en las dos técnicas de digestibilidad, mientras que en la DER de la FDN, se observó un dato más alto $(P<0,05)$ con la técnica in situ (Tablas $11,12$ y 13$)$.

Tabla 8. Digestibilidad in situ Vs in vitro yopo (Anadenanthera (Piptadenia) peregrina)

\begin{tabular}{|c|c|c|c|c|c|c||}
\hline \multirow{2}{*}{ Horas } & \multicolumn{2}{|c|}{ Digestibilidad M.S. (\%) } & \multicolumn{2}{c|}{ Digestibilidad FDN (\%) } & \multicolumn{2}{|c|}{ Digestibilidad Proteina (\%) } \\
\cline { 2 - 7 } & In Situ & In Vitro & In Situ & In Vitro & In Situ & In vitro \\
\hline 6 & $28,350^{\mathrm{a}}$ & $27,151^{\mathrm{a}}$ & $35,384^{\mathrm{b}}$ & $29,641^{\mathrm{a}}$ & $30,439^{\mathrm{b}}$ & $25,679^{\mathrm{a}}$ \\
\hline 12 & $31,542^{\mathrm{a}}$ & $33,050^{\mathrm{b}}$ & $36,496^{\mathrm{b}}$ & $31,144^{\mathrm{a}}$ & $36,279^{\mathrm{b}}$ & $31,808^{\mathrm{a}}$ \\
\hline 24 & $34,603^{\mathrm{a}}$ & $36,173^{\mathrm{b}}$ & $37,588^{\mathrm{b}}$ & $32,382^{\mathrm{a}}$ & $39,511^{\mathrm{b}}$ & $35,326^{\mathrm{a}}$ \\
\hline 48 & $42,525^{\mathrm{b}}$ & $40,552^{\mathrm{a}}$ & $39,600^{\mathrm{b}}$ & $33,570^{\mathrm{a}}$ & $47,703^{\mathrm{b}}$ & $36,985^{\mathrm{a}}$ \\
\hline 72 & $50,293^{\mathrm{a}}$ & $48,976^{\mathrm{a}}$ & $49,468^{\mathrm{b}}$ & $35,529^{\mathrm{a}}$ & $58,910^{\mathrm{a}}$ & $54,512^{\mathrm{a}}$ \\
\hline
\end{tabular}

* Materia Seca. ${ }^{* *}$ Fibra Detergente Neutro. Letras distintas en las columnas de cada nutriente in situ e in vitro son diferentes $(\mathrm{P}<0,05)$. 
De igual manera las digestibilidades in situ de la MS, FDN y NT del palo de cruz fueron mayores $(P<0,05)$ que las in vitro (Tabla 9 - Figura 7$)$. La cinética de la degradación fue diferente en todos los nutrientes, encontrándose que la DER de la MS, FDN y NT fue mayor $(\mathrm{P}<0,05)$ mediante la técnica in situ (Tablas 11, 12 y 13). Igualmente, las digestibilidades in situ de la MS, FDN y NT del matarratón fueron mayores $(\mathrm{P}<0,05)$ que las in vitro (Tabla 10 Figura 9). La cinética de la degradación fue diferente en todos los nutrientes, al igual que en el caso anterior la DER de la MS, FDN NT fue mayor $(P<0,05)$ en la estimación mediante la técnica in situ (Tablas 11, 12 y 13).

Tabla 9. Digestibilidad in situ Vs in vitro palo de cruz (Brownea ariza)

\begin{tabular}{|c|c|c|c|c|c|c|}
\hline \multirow{2}{*}{ Horas } & \multicolumn{2}{|c|}{ Digestibilidad M.S. (\%) } & \multicolumn{2}{c|}{ Digestibilidad FDN (\%) } & \multicolumn{2}{c|}{ Digestibilidad Proteina (\%) } \\
\cline { 2 - 7 } & In Situ & In Vitro & In Situ & In Vitro & In Situ & In vitro \\
\hline 6 & $26,987^{\mathrm{b}}$ & $23,477^{\mathrm{a}}$ & $24,692^{\mathrm{b}}$ & $19,684^{\mathrm{a}}$ & $51,905^{\mathrm{b}}$ & $21,093^{\mathrm{a}}$ \\
\hline 12 & $29,503^{\mathrm{b}}$ & $26,381^{\mathrm{a}}$ & $30,042^{\mathrm{b}}$ & $20,820^{\mathrm{a}}$ & $54,278^{\mathrm{b}}$ & $24,683^{\mathrm{a}}$ \\
\hline 24 & $32,451^{\mathrm{b}}$ & $29,504^{\mathrm{a}}$ & $31,530^{\mathrm{b}}$ & $21,990^{\mathrm{a}}$ & $56,059^{\mathrm{b}}$ & $28,901^{\mathrm{a}}$ \\
\hline 48 & $33,894^{\mathrm{b}}$ & $31,254^{\mathrm{a}}$ & $34,901^{\mathrm{b}}$ & $23,424^{\mathrm{a}}$ & $59,023^{\mathrm{b}}$ & $36,579^{\mathrm{a}}$ \\
\hline 72 & $37,217^{\mathrm{b}}$ & $33,389^{\mathrm{a}}$ & $39,746^{\mathrm{b}}$ & $26,649^{\mathrm{a}}$ & $62,087^{\mathrm{b}}$ & $41,678^{\mathrm{a}}$ \\
\hline
\end{tabular}

* Materia Seca. ${ }^{* *}$ Fibra Detergente Neutro. Letras distintas en las columnas de cada nutriente in situ e in vitro son diferentes $(\mathrm{P}<0,05)$.

Tabla 10. Digestibilidad in situ Vs in vitro matarratón (Gliricidia sepium)

\begin{tabular}{|c|c|c|c|c|c|c|}
\hline \multirow{2}{*}{ Horas } & \multicolumn{2}{|c|}{ Digestibilidad M.S. (\%) } & \multicolumn{2}{|c|}{ Digestibilidad FDN (\%) } & \multicolumn{2}{|c|}{ Digestibilidad Proteina (\%) } \\
\cline { 2 - 7 } & In Situ & In Vitro & In Situ & In Vitro & In Situ & In vitro \\
\hline 6 & $23,898^{\mathrm{b}}$ & $8,347^{\mathrm{a}}$ & $34,562^{\mathrm{b}}$ & $23,959^{\mathrm{a}}$ & $32,425^{\mathrm{b}}$ & $13,311^{\mathrm{a}}$ \\
\hline 12 & $31,195^{\mathrm{b}}$ & $12,581^{\mathrm{a}}$ & $37,010^{\mathrm{b}}$ & $26,373^{\mathrm{a}}$ & $39,634^{\mathrm{b}}$ & $15,381^{\mathrm{a}}$ \\
\hline 24 & $39,984^{\mathrm{b}}$ & $14,981^{\mathrm{a}}$ & $42,310^{\mathrm{b}}$ & $28,449^{\mathrm{a}}$ & $45,140^{\mathrm{b}}$ & $17,665^{\mathrm{a}}$ \\
\hline 48 & $47,908^{\mathrm{b}}$ & $17,652^{\mathrm{a}}$ & $43,337^{\mathrm{b}}$ & $29,840^{\mathrm{a}}$ & $53,402^{\mathrm{b}}$ & $22,916^{\mathrm{a}}$ \\
\hline 72 & $55,803^{\mathrm{b}}$ & $21,191^{\mathrm{a}}$ & $56,352^{\mathrm{b}}$ & $30,936^{\mathrm{a}}$ & $61,556^{\mathrm{b}}$ & $31,634^{\mathrm{a}}$ \\
\hline
\end{tabular}

* Materia Seca. ${ }^{* *}$ Fibra Detergente Neutro. Letras distintas en las columnas de cada nutriente in situ e in vitro son diferentes $(\mathrm{P}<0,05)$.

En la MS se encontró una tasa de degradación más alta mediante la técnica in situ que con la in vitro (Tabla 11). Se observaron valores de DER más altos en el pasto amargo, botón de oro, casco de vaca, yopo, palo de cruz, cayeno y matarratón, mientras que en los forrajes kingrass, veranera y acacia roja la DER fue más alta mediante la técnica in vitro.

Así mismo, en la FDN se encontró una tasa de degradación mayor mediante la técnica in situ (Tabla 12). Se encontraron valores de DER más altos el botón de oro, casco de vaca, veranera, yopo, palo de cruz y matarratón; para los forrajes pasto amargo kingrass, cayeno y acacia roja la DER fue más alta mediante la técnica in vitro. Igualmente en el NT la tasa de 
degradación fue más alta con la técnica in situ (Tabla 13.). Se encontraron valores de DER mas altos el botón de oro, yopo, palo de cruz, cayeno, matarratón y acacia roja, mientras que para los forrajes pasto amargo kingrass, casco de vaca, veranera la DER fue más alta mediante la técnica in vitro.

Tabla 11. Parámetros de cinética ruminal de la DMS

\begin{tabular}{|c|c|c|c|c|c|}
\hline \multicolumn{6}{|c|}{ CINETICA RUMINAL DEGRADABILIDAD MATERIA SECA } \\
\hline FORRAJE & \begin{tabular}{|c|} 
Tecnica de \\
Digestibilidad
\end{tabular} & $A(\%)$ & B (\%) & \begin{tabular}{|c|} 
C (Fraccion \\
/ Hora)
\end{tabular} & DE (\%) \\
\hline \multirow{2}{*}{ Brachiaria decumbens } & In Situ & 10,179 & 41,681 & 0,038 & $48,598^{a}$ \\
\hline & In Vitro & 13,336 & 35,185 & 0,142 & $48,519^{a}$ \\
\hline \multirow{2}{*}{ Pennisetum hibridum } & In Situ & 6,731 & 31,587 & 0,042 & $36,460^{a}$ \\
\hline & In Vitro & 7,964 & 31,328 & 0,102 & $39,266^{a}$ \\
\hline \multirow{2}{*}{ Tithonia diversifolia } & In Situ & 7,618 & 53,721 & 0,049 & $59,525^{b}$ \\
\hline & In Vitro & 11,746 & 32,763 & 0,108 & $44,491^{a}$ \\
\hline \multirow{2}{*}{ Bahuinia variegata } & In Situ & 11,117 & 20,625 & 0,235 & $31,742^{b}$ \\
\hline & In Vitro & 4,785 & 23,990 & 0,105 & $28,760^{a}$ \\
\hline \multirow{2}{*}{ Cratylia argentea } & In Situ & 20,747 & 50,186 & 0,168 & $70,932^{a}$ \\
\hline & In Vitro & 36,174 & 47,778 & 0,376 & $83,951^{b}$ \\
\hline \multirow{2}{*}{$\begin{array}{c}\text { Anadenanthera } \\
\text { (Piptadenia) peregrina }\end{array}$} & In Situ & 10,826 & 32,765 & 0,126 & $43,586^{\mathrm{a}}$ \\
\hline & In Vitro & 10,401 & 32,222 & 0,139 & $42,622^{a}$ \\
\hline \multirow{2}{*}{ Brownea ariza } & In Situ & 11,945 & 22,172 & 0,232 & $34,117^{b}$ \\
\hline & In Vitro & 10,611 & 20,523 & 0,206 & $31,134^{a}$ \\
\hline \multirow{2}{*}{ Hibiscus rosa-sinensis } & In Situ & 13,935 & 76,042 & 0,035 & $82,602^{b}$ \\
\hline & In Vitro & 10,795 & 42,486 & 0,092 & $53,212^{a}$ \\
\hline \multirow{2}{*}{ Gliricidia sepium } & In Situ & 9,923 & 41,826 & 0,078 & $51,560^{b}$ \\
\hline & In Vitro & 8,347 & 21,191 & 0,294 & $29,538^{a}$ \\
\hline \multirow{2}{*}{ Delonix regia } & In Situ & 10,055 & 28,445 & 0,124 & $38,495^{a}$ \\
\hline & In Vitro & 19,226 & 23,653 & 0,465 & $42,879^{a}$ \\
\hline
\end{tabular}

DMS = Degradabilidad Materia Seca. $\mathrm{A}=$ Fracción rápidamente soluble. $\mathrm{B}=$ Fracción insoluble pero potencialmente degradable. $\mathrm{C}=$ Tasa de degradación de la fracción $\mathrm{B}$. DE = Degradabilidad Efectiva Ruminal. Letras distintas en el mismo tiempo de incubación son diferentes $(\mathrm{P}<0,05)$.

El nitrógeno amoniacal fue más bajo en el líquido ruminal del animal de mejor condición corporal, en donde se incubaron las especies forrajeras de mayor contenido proteico, botón de oro, veranera, matarratón, cayeno y acacia roja, excepto a las 6 horas de incubación. El pH fue más alto en el líquido de este mismo animal, excepto a las 12 horas de incubación. (Tabla 14).

\section{DISCUSIÓN}

En la digestibilidad de la MS de los forrajes B. variegata, C. argentea, B. ariza, H. rosasinensis y G. sepium, que son el $50 \%$ (Figura 11) de las especies estudiadas, presentaron 
una diferencia $(P<0,05)$ del $100 \%$, es decir que las dos técnicas no son compatibles para este nutriente en estos forrajes. Así mismo, D. regia que representa el $10 \%$ de los forrajes estudiados, obtuvo una diferencia $(P<0,05)$ del $80 \%$, lo cual significa que en esta especie hay muy poca compatibilidad entre estas dos técnicas. Por otro lado los forrajes $B$. decumbens, P. purpureum, T. diversifolia y $A$. (Piptadenia) peregrina, que corresponden al $40 \%$ de especies estudiadas mostraron una diferencia $(P<0,05)$ del $60 \%$, quedando un $40 \%$ de similaridad entre las dos técnicas, por lo tanto en estos forrajes es posible seguir investigando en las pruebas hasta determinar una compatibilidad o diferencia total entre las dos técnicas de digestibilidad para este nutriente.

Tabla 12. Parámetros de cinética ruminal de la DFDN

\begin{tabular}{|c|c|c|c|c|c|}
\hline \multicolumn{6}{|c|}{ CINETICA RUMINAL DEGRADABILIDAD FIBRA DETERGENTE NEUTRO } \\
\hline \multirow{2}{*}{ FORRAJE } & $\begin{array}{c}\text { Tecnica de } \\
\text { Digestibilidad }\end{array}$ & A (\%) & B (\%) & $\begin{array}{c}\text { C (Fraccion } \\
\text { / Hora) }\end{array}$ & DE (\%) \\
\hline \multirow{2}{*}{ Brachiaria decumbens } & In Situ & 20,848 & 26,975 & 0,388 & $47,823^{\mathrm{a}}$ \\
\cline { 2 - 6 } & In Vitro & 24,657 & 30,623 & 0,457 & $55,280^{\mathrm{b}}$ \\
\hline \multirow{2}{*}{ Pennisetum hibridum } & In Situ & 13,190 & 20,500 & 0,289 & $33,690^{\mathrm{a}}$ \\
\cline { 2 - 7 } & In Vitro & 14,501 & 20,764 & 0,382 & $35,265^{\mathrm{b}}$ \\
\hline \multirow{3}{*}{ Tithonia diversifolia } & In Situ & 19,009 & 23,368 & 0,467 & $42,377^{\mathrm{b}}$ \\
\cline { 2 - 6 } & In Vitro & 6,905 & 18,071 & 0,172 & $24,976^{\mathrm{a}}$ \\
\hline \multirow{2}{*}{ Bahuinia variegata } & In Situ & 9,731 & 26,843 & 0,161 & $36,574^{\mathrm{b}}$ \\
\cline { 2 - 7 } & In Vitro & 9,373 & 19,340 & 0,254 & $28,713^{\mathrm{a}}$ \\
\hline \multirow{2}{*}{ Cratylia argentea } & In Situ & 33,131 & 34,154 & 0,756 & $67,285^{\mathrm{b}}$ \\
\cline { 2 - 7 } & In Vitro & 31,219 & 35,095 & 0,505 & $66,314^{\mathrm{a}}$ \\
\hline \multirow{2}{*}{ Anadenanthera } & In Situ & 13,663 & 27,744 & 0,287 & $41,407^{\mathrm{b}}$ \\
\cline { 2 - 7 } (Piptadenia) peregrina & In Vitro & 13,754 & 19,640 & 0,346 & $33,393^{\mathrm{a}}$ \\
\hline \multirow{2}{*}{ Brownea ariza } & In Situ & 10,178 & 25,229 & 0,178 & $35,408^{\mathrm{b}}$ \\
\cline { 2 - 7 } & In Vitro & 8,302 & 15,266 & 0,270 & $23,568^{\mathrm{a}}$ \\
\hline \multirow{2}{*}{ Hibiscus rosa-sinensis } & In Situ & 22,192 & 25,643 & 0,486 & $47,836^{\mathrm{a}}$ \\
\cline { 2 - 7 } & In Vitro & 23,229 & 28,390 & 0,436 & $51,619^{\mathrm{b}}$ \\
\hline \multirow{2}{*}{ Gliricidia sepium } & In Situ & 12,629 & 34,417 & 0,177 & $47,046^{\mathrm{b}}$ \\
\cline { 2 - 6 } & In Vitro & 11,219 & 18,194 & 0,259 & $29,413^{\mathrm{a}}$ \\
\hline \multirow{2}{*}{ Delonix regia } & In Situ & 14,441 & 19,786 & 0,369 & $34,227^{\mathrm{a}}$ \\
\cline { 2 - 6 } & In Vitro & 14,142 & 21,414 & 0,323 & $35,556^{\mathrm{b}}$ \\
\hline
\end{tabular}

DMS = Degradabilidad Materia Seca. DFDN= Degradabilidad fibra detergente neutro. $A=$ Fracción rápidamente soluble. $B=$ Fracción insoluble pero potencialmente degradable. $C=$ Tasa de degradación de la fracción $B$. DE = Degradabilidad Efectiva Ruminal. Letras distintas en el mismo tiempo de incubación son diferentes $(\mathrm{P}<0,05)$.

En la digestibilidad de la FDN de los forrajes $B$. decumbens, $T$. diversifolia, $C$. argéntea, $A$. (Piptadenia) peregrina, B. ariza, H. rosa-sinensis y G. sepium, que son el 70\% (Figura 12) de las especies estudiadas, presentaron una diferencia $(P<0,05)$ del $100 \%$, es decir que las dos técnicas no son compatibles para este nutriente en estos forrajes. Así mismo, P. purpureum y 
B. variegata, que representan el $20 \%$ de los forrajes estudiados, obtuvieron una diferencia $(\mathrm{P}<0,05)$ del $80 \%$, lo cual significa que en estas especies hay poca compatibilidad entre estas dos técnicas. Por otro lado, D. regia forraje que corresponde al $10 \%$ de las especies estudiadas mostró una diferencia $(\mathrm{P}<0,05)$ del 60\%, quedando un $40 \%$ de similaridad entre las dos técnicas, por lo tanto en este forraje es posible seguir investigando en las pruebas hasta determinar una compatibilidad o diferencia total entre las dos técnicas de digestibilidad para este nutriente.

Tabla 13. Parámetros de cinética ruminal de la DNT

\begin{tabular}{|c|c|c|c|c|c|}
\hline \multicolumn{6}{|c|}{ CINETICA RUMINAL DEGRADABILIDAD NITROGENO TOTAL } \\
\hline \multirow{2}{*}{ FORRAJE } & $\begin{array}{c}\text { Tecnica de } \\
\text { Digestibilidad }\end{array}$ & $\mathrm{A}(\%)$ & $\mathrm{B}(\%)$ & $\begin{array}{c}\text { ( Fraccion } \\
\text { / Hora) }\end{array}$ & DE (\%) \\
\hline \multirow{2}{*}{ Brachiaria decumbens } & In Situ & 12,988 & 52,745 & 0,084 & $65,580^{\circ}$ \\
\cline { 2 - 6 } & In Vitro & 14,642 & 51,340 & 0,133 & $65,978^{\circ}$ \\
\hline \multirow{2}{*}{ Pennisetum hibridum } & In Situ & 9,271 & 40,529 & 0,089 & $49,718^{\circ}$ \\
\cline { 2 - 6 } & In Vitro & 17,625 & 33,100 & 0,244 & $50,725^{\circ}$ \\
\hline \multirow{2}{*}{ Tithonia diversifolia } & In Situ & 15,069 & 47,600 & 0,102 & $62,628^{\circ}$ \\
\cline { 2 - 6 } & In Vitro & 17,262 & 37,233 & 0,184 & $54,495^{\circ}$ \\
\hline \multirow{2}{*}{ Bahuinia variegata } & In Situ & 8,489 & 29,788 & 0,114 & $38,267^{\circ}$ \\
\cline { 2 - 6 } & In Vitro & 10,888 & 27,793 & 0,155 & $38,681^{\circ}$ \\
\hline \multirow{2}{*}{ Cratylia argentea } & In Situ & 11,878 & 30,044 & 0,176 & $41,922^{\circ}$ \\
\cline { 2 - 6 } & In Vitro & 38,055 & 48,055 & 0,408 & $86,110^{\mathrm{b}}$ \\
\hline \multirow{2}{*}{ Anadenanthera } & In Situ & 11,111 & 39,579 & 0,113 & $50,675^{\circ}$ \\
\cline { 2 - 6 } (Piptadenia) peregrina & In Vitro & 7,578 & 36,408 & 0,112 & $43,972^{\circ}$ \\
\hline \multirow{2}{*}{ Brownea ariza } & In Situ & 24,044 & 34,222 & 0,350 & $58,267^{\mathrm{b}}$ \\
\cline { 2 - 6 } & In Vitro & 8,628 & 29,208 & 0,094 & $37,793^{\circ}$ \\
\hline \multirow{2}{*}{ Hibiscus rosa-sinensis } & In Situ & 14,325 & 68,213 & 0,057 & $81,212^{\mathrm{b}}$ \\
\cline { 2 - 6 } & In Vitro & 14,548 & 39,533 & 0,150 & $54,080^{\circ}$ \\
\hline \multirow{2}{*}{ Gliricidia sepium } & In Situ & 13,032 & 42,160 & 0,115 & $55,177^{\mathrm{b}}$ \\
\cline { 2 - 6 } & In Vitro & 13,311 & 31,634 & 0,439 & $44,945^{\circ}$ \\
\hline \multirow{2}{*}{ Delonix regia } & In Situ & 10,123 & 32,568 & 0,120 & $42,683^{\mathrm{b}}$ \\
\cline { 2 - 6 } & In Vitro & 11,013 & 22,363 & 0,214 & $33,376^{\circ}$ \\
\hline
\end{tabular}

DMS = Degradabilidad Materia Seca. $\mathrm{DNT}=$ Degradabilidad nitrógeno total $. \mathrm{A}=$ Fracción rápidamente soluble. $\mathrm{B}=$ Fracción insoluble pero potencialmente degradable $\mathrm{C}=$ Tasa de degradación de la fracción $\mathrm{B}$. DE = Degradabilidad Efectiva Ruminal. Letras distintas en el mismo tiempo de incubación son diferentes $(\mathrm{P}<0,05)$.

Tabla 14. Nitrógeno Amoniacal y pH en la prueba in situ

\begin{tabular}{|c|c|c|c|}
\hline \multicolumn{4}{|c|}{ MAYOR PROTEINA * } \\
\hline $\begin{array}{c}\text { HORA } \\
\text { DIGESTIBILIDAD }\end{array}$ & pH & $\begin{array}{c}\text { TEMPERATURA } \\
\left(\mathbf{C}^{\circ}\right)\end{array}$ & $\begin{array}{c}\text { NITROGENO } \\
\text { AMONIACAL }\end{array}$ \\
\hline 0 & 6,6 & 38 & 29,12 \\
\hline 6 & 6,47 & 37,9 & 43,12 \\
\hline 12 & 6,63 & 37,8 & 43,12 \\
\hline \multicolumn{4}{|c|}{ MENOR PROTEINA ** } \\
\hline $\begin{array}{c}\text { HORA } \\
\text { DIGESTIBILIDAD }\end{array}$ & pH & $\begin{array}{c}\text { TEMPERATURA } \\
\left(\mathbf{C}^{\circ}\right)\end{array}$ & $\begin{array}{c}\text { NITROGENO } \\
\text { AMONIACAL }\end{array}$ \\
\hline 0 & 6,5 & 37 & 32,2 \\
\hline 6 & 6,4 & 38 & 71,12 \\
\hline 12 & 6,77 & 37,7 & 40,88 \\
\hline
\end{tabular}

${ }^{*}$ Forrajes incubados: Tithonia diversifolia, Cratylia argéntea, Gliricidia sepium, Hibiscus rosa-sinensis y Delonix regia. ${ }^{* *}$ Forrajes incubados: Brachiaria decumbens, Pennisetum purpureum, Bauhinia variegata, Anadenanthera (Piptadenia) peregrina y Brownea ariza. 


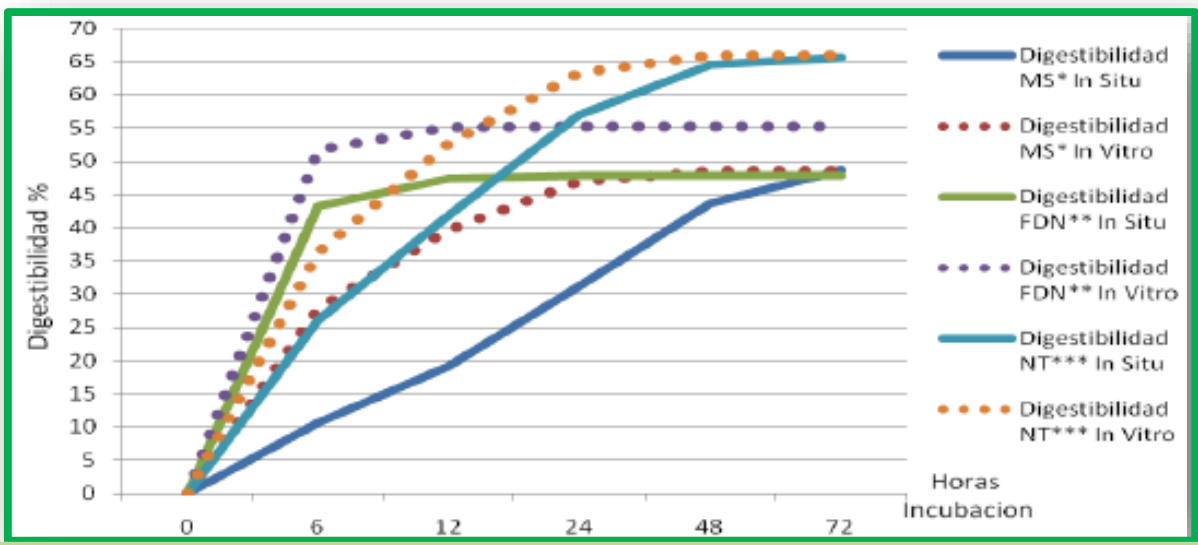

Figura 1. Curva de degradación pasto amargo (Brachiaria

* Materia Seca. ${ }^{* *}$ Fibra Detergente Neutro ***Nitrógeno Total

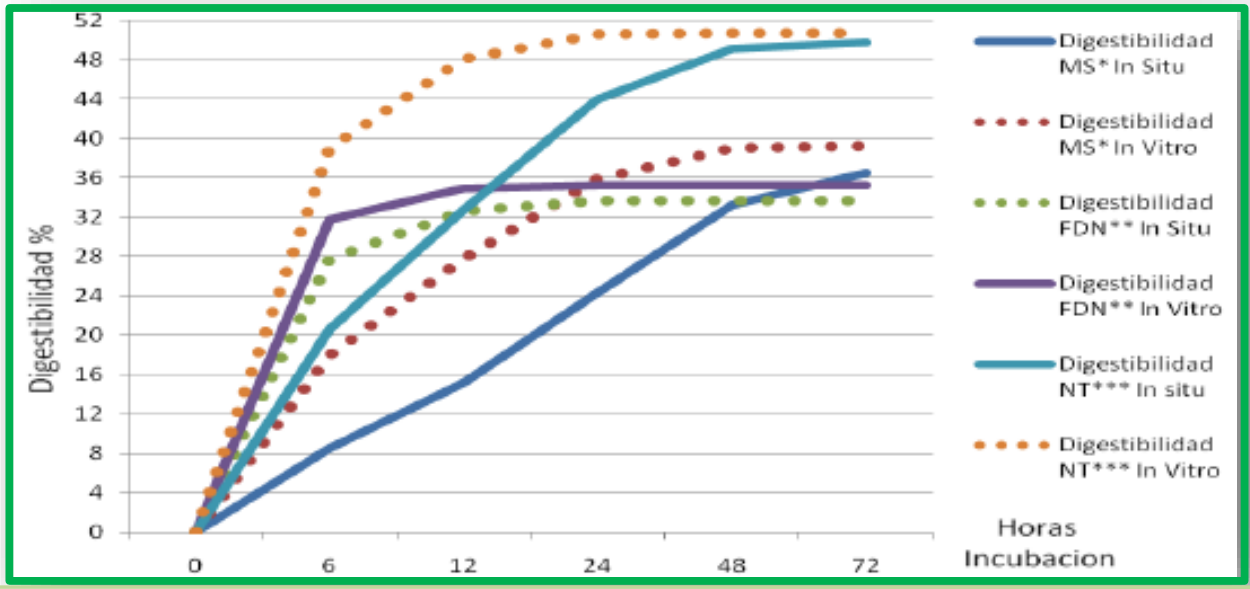

Figura 2. Curva de degradación kingrass (Pennisetum purpureum)

* Materia Seca. ${ }^{* *}$ Fibra Detergente Neutro ***Nitrógeno Total

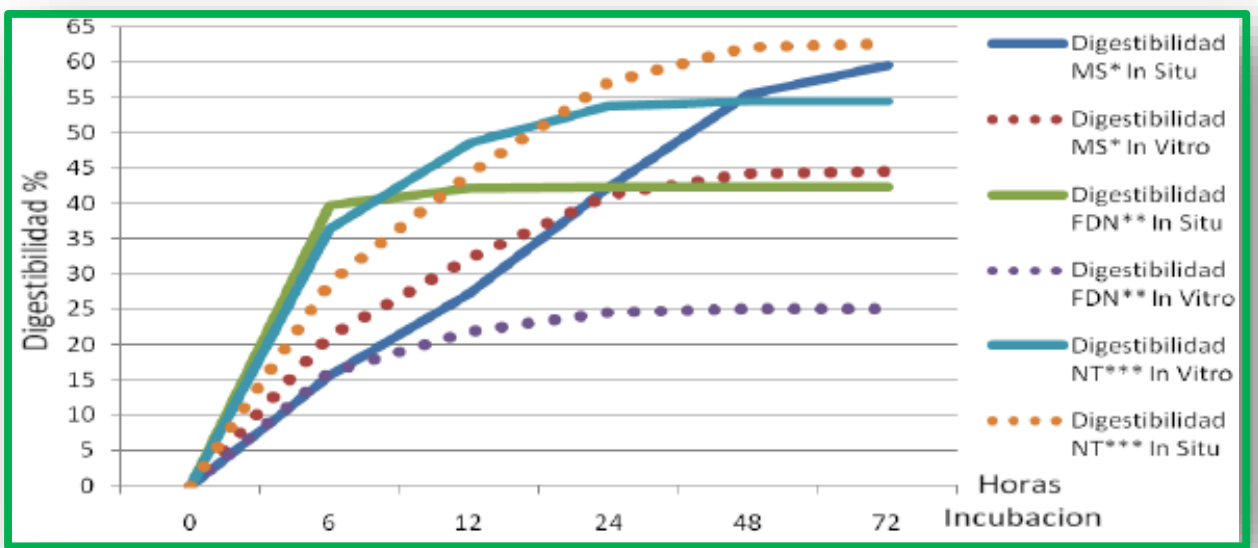

Figura 3. Curva de degradación botón de oro (Tithonia diversifolia)

* Materia Seca. ${ }^{* *}$ Fibra Detergente Neutro ***Nitrógeno Total 


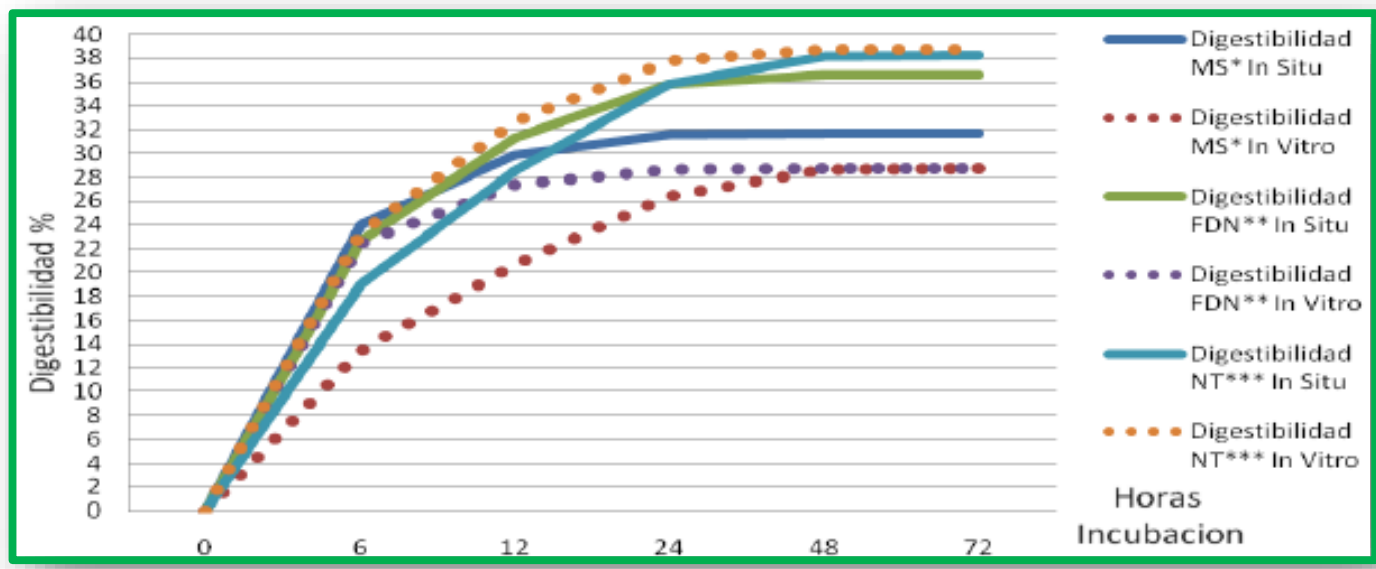

Figura 4. Curva de degradación casco de vaca (Bauhinia variegata)

* Materia Seca. ${ }^{* *}$ Fibra Detergente Neutro ***Nitrógeno Total

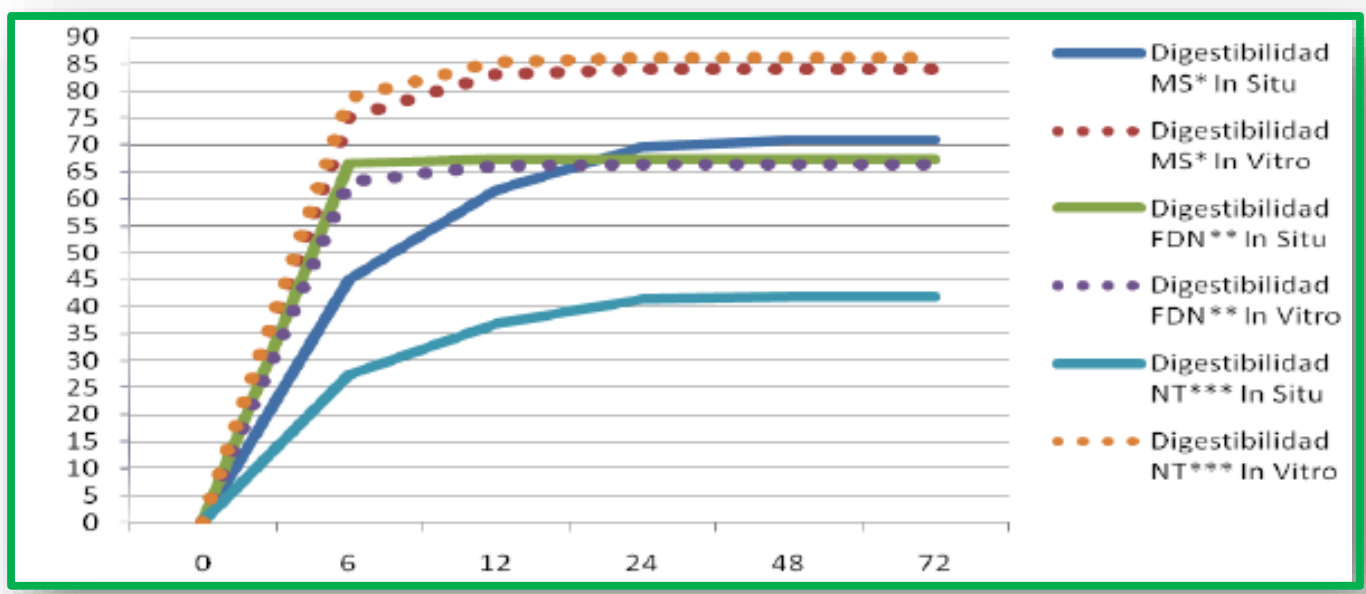

Figura 5. Curva de degradación veranera (Cratylia argentea)

* Materia Seca. ${ }^{* *}$ Fibra Detergente Neutro ***Nitrógeno Total

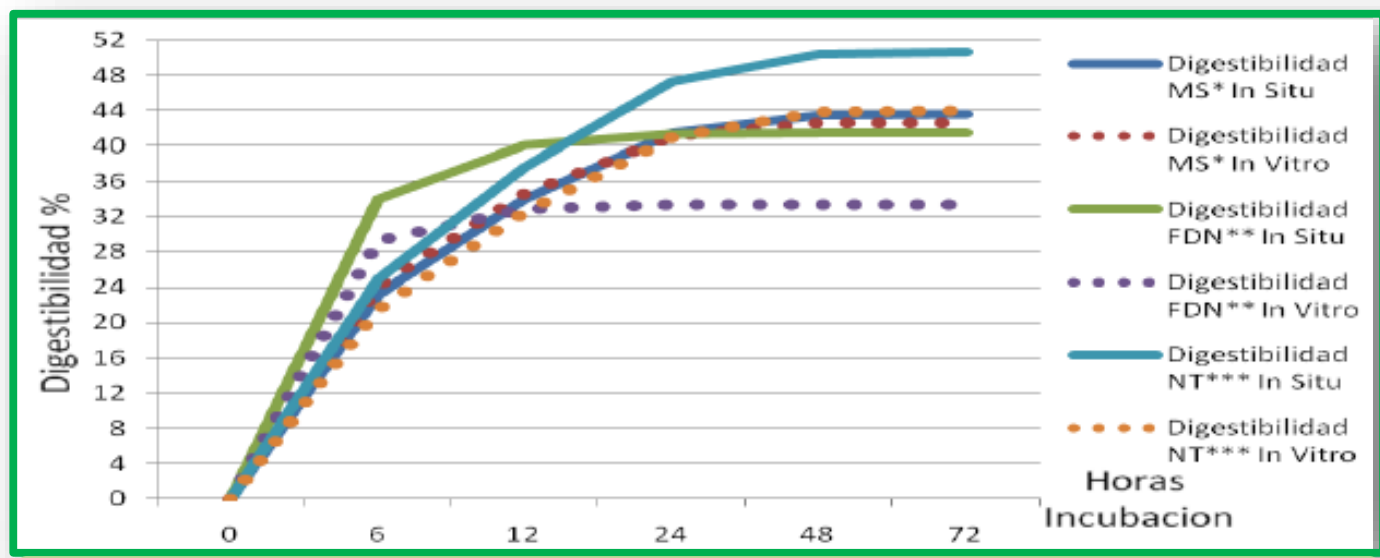

Figura 6. Curva de degradación yopo Piptadenia peregrina

${ }^{\star}$ Materia Seca. ${ }^{* \star}$ Fibra Detergente Neutro ${ }^{* \star *}$ Nitrógeno Total 


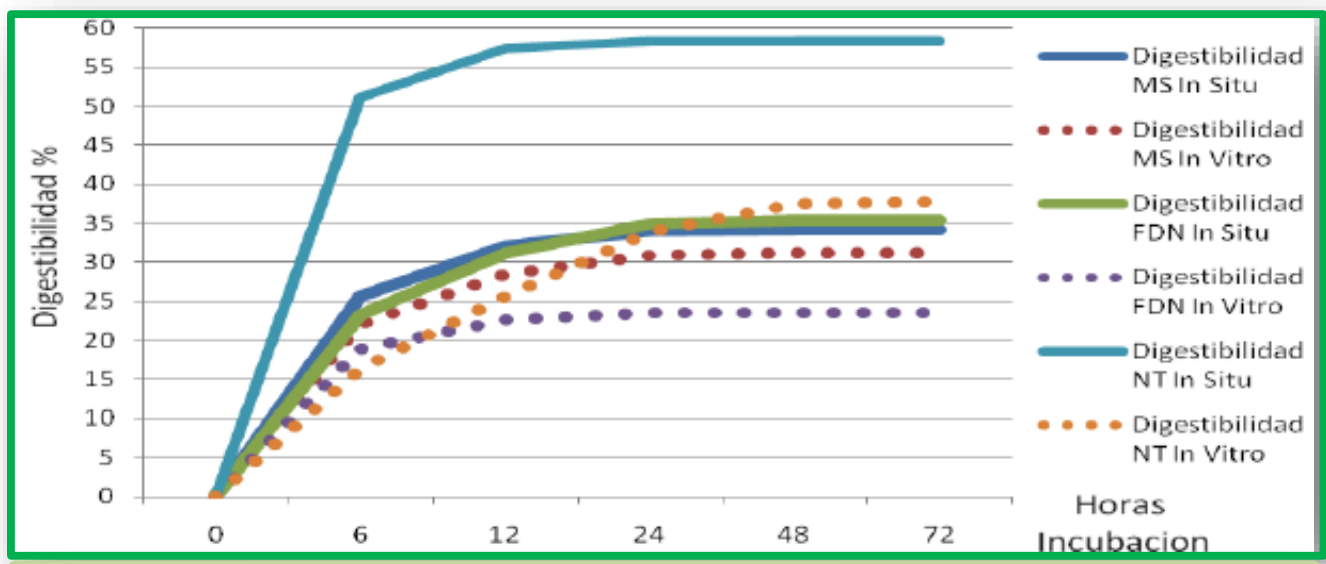

Figura 7. Curva de degradación palo de cruz (Brownea ariza)

${ }^{*}$ Materia Seca. ${ }^{* *}$ Fibra Detergente Neutro ${ }^{* * *}$ Nitrógeno Total

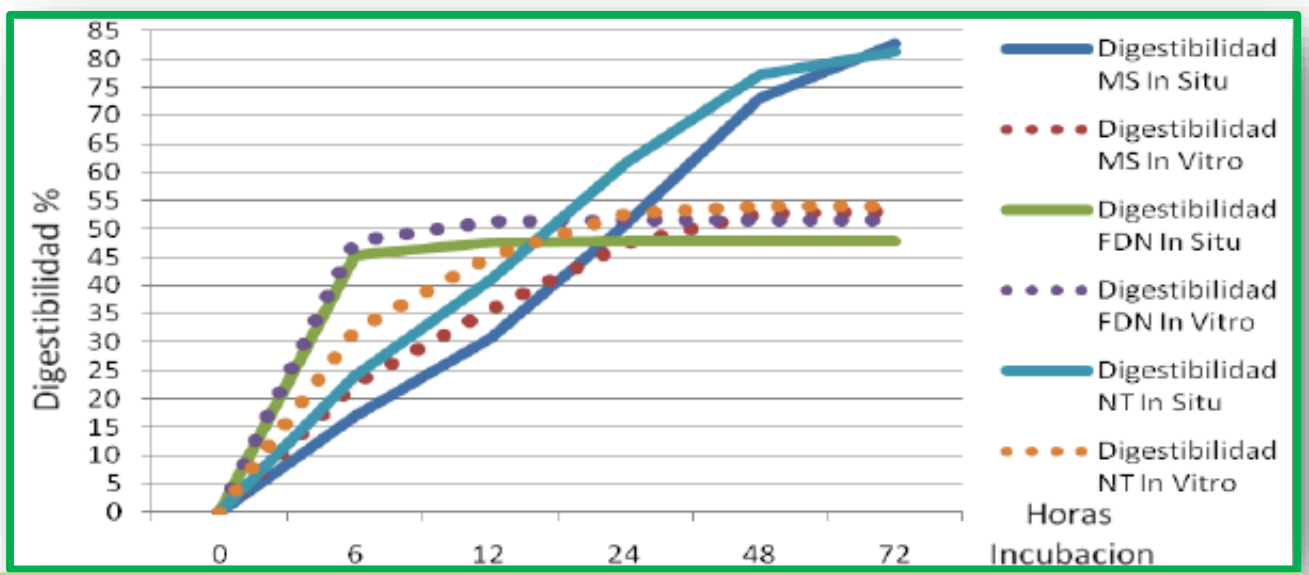

Figura 8. Curva de degradación cayeno (Hibiscus rosa-sinensis)

${ }^{*}$ Materia Seca. ${ }^{* *}$ Fibra Detergente Neutro ***Nitrógeno Total

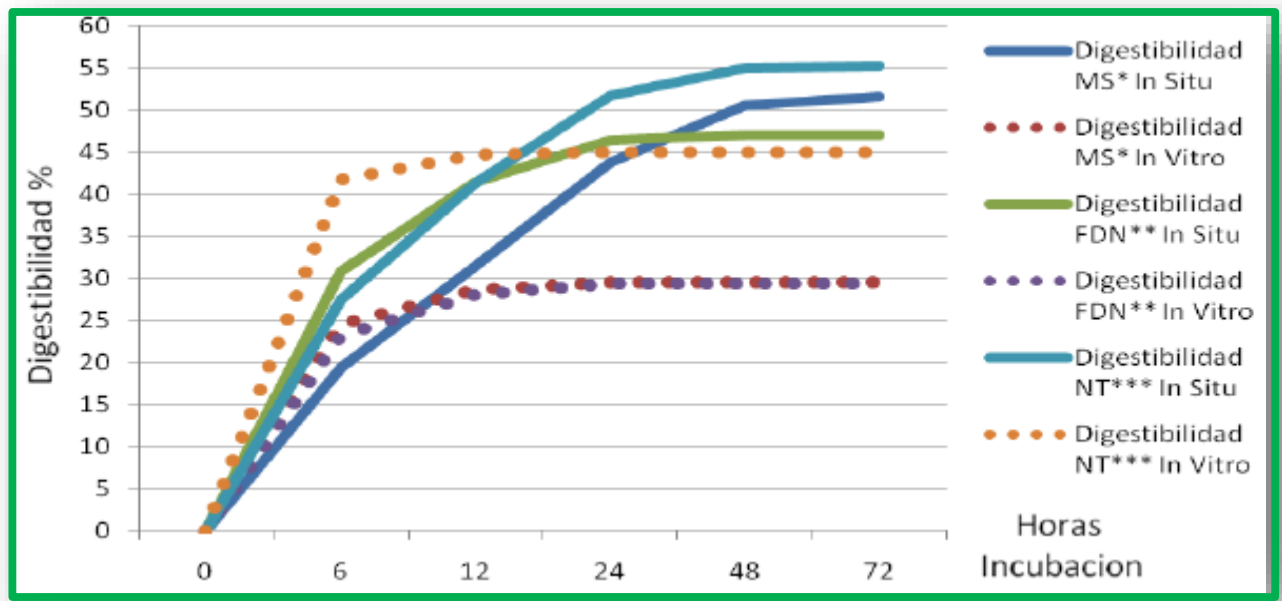

Figura 9. Curva de degradación matarratón (Gliricidia sepium)

* Materia Seca. ${ }^{* *}$ Fibra Detergente Neutro ***Nitrógeno Total 


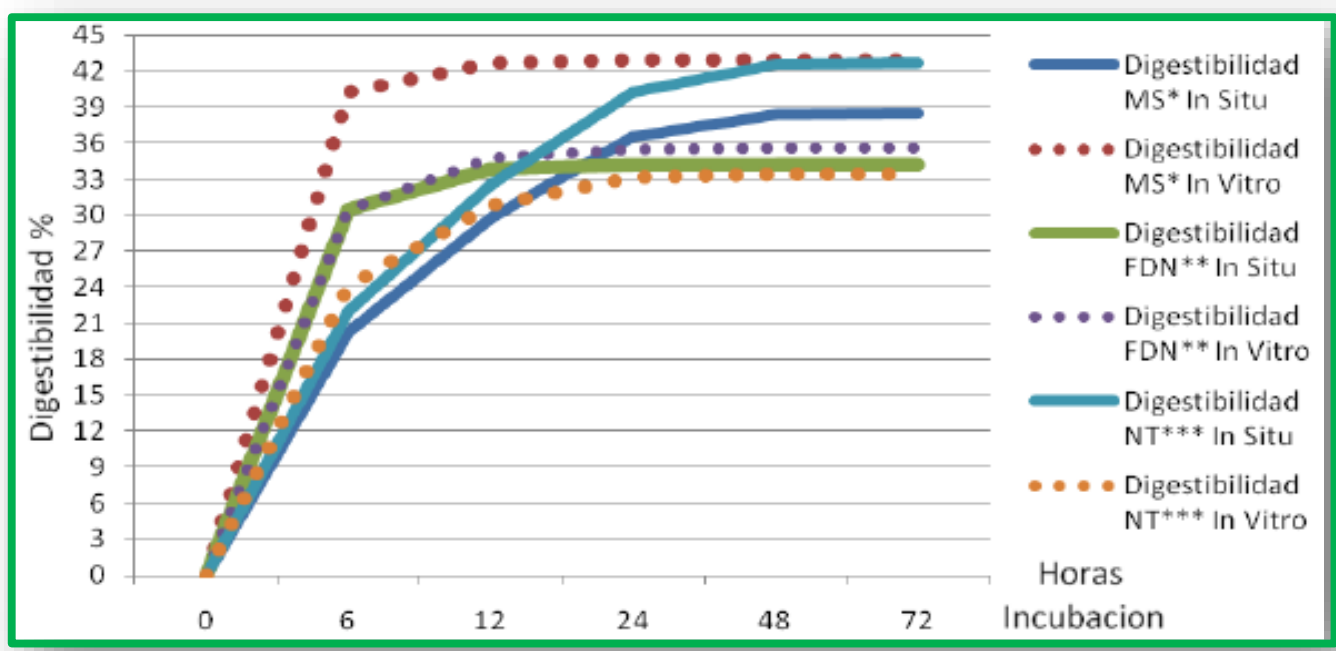

Figura 10. Curva de degradación acacia roja (Delonix regia)

${ }^{\star}$ Materia Seca. ${ }^{\star *}$ Fibra Detergente Neutro ${ }^{* \star *}$ Nitrógeno Total

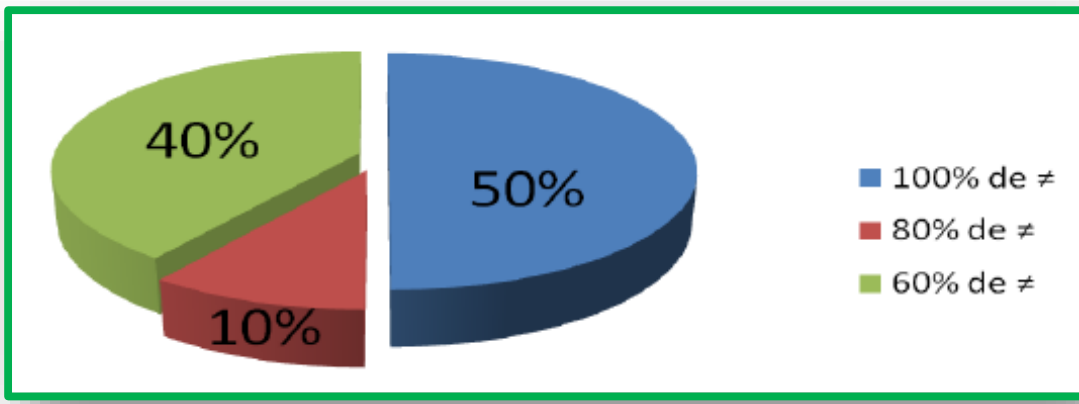

Figura 11. Distribución de la diferencia estadística materia seca entre las dos técnicas. Diferencia $(\neq)$ Estadística $P<0,0.5$

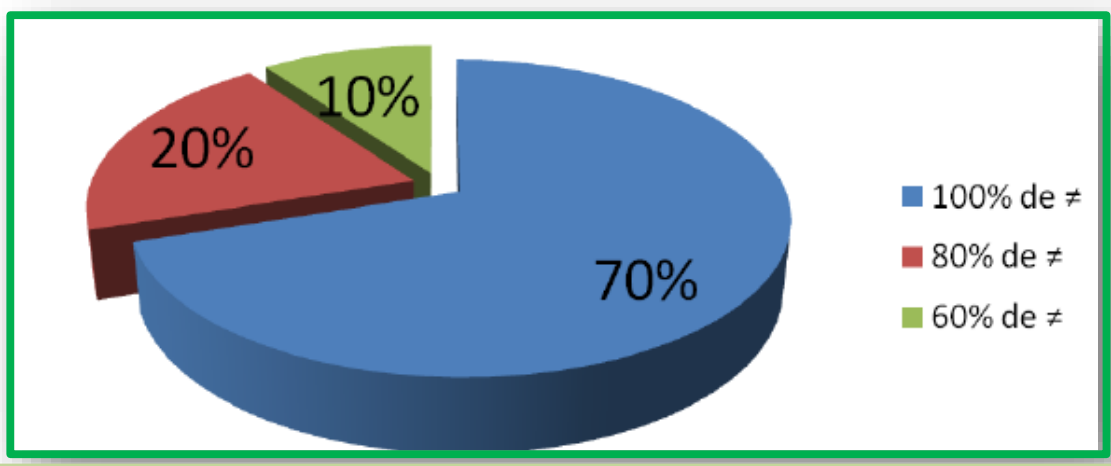

Figura 12. Distribución de la diferencia estadística fibra detergente neutro, entre las dos técnicas Diferencia $(\neq)$ Estadística $(P<0,0.5)$

En la digestibilidad del NT de los forrajes C. argéntea, B. ariza y G. sepium, que son el 30\% (Figura 13) de las especies estudiadas, presentaron una diferencia $(P<0,05)$ del $100 \%$, es 
decir que las dos técnicas no son compatibles para este nutriente en estos forrajes. Así mismo, B. decumbens, P. purpureum, B. variegata, Piptadenia peregrina y Delonix regia, que representan el $50 \%$ de los forrajes estudiados, obtuvieron una diferencia $(P<0,05)$ del $80 \%$, lo cual significa que en estas especies hay muy poca compatibilidad entre estas dos técnicas. Por otro lado los forrajes $T$. diversifolia e $H$. rosa-sinensis, que corresponden al $20 \%$ las de especies estudiadas mostraron una diferencia $(P<0,05)$ del $60 \%$, quedando un $40 \%$ de similaridad entre las dos técnicas, por lo tanto en estos forrajes es posible seguir investigando en las pruebas hasta determinar una compatibilidad o diferencia total entre las dos técnicas de digestibilidad para este nutriente.

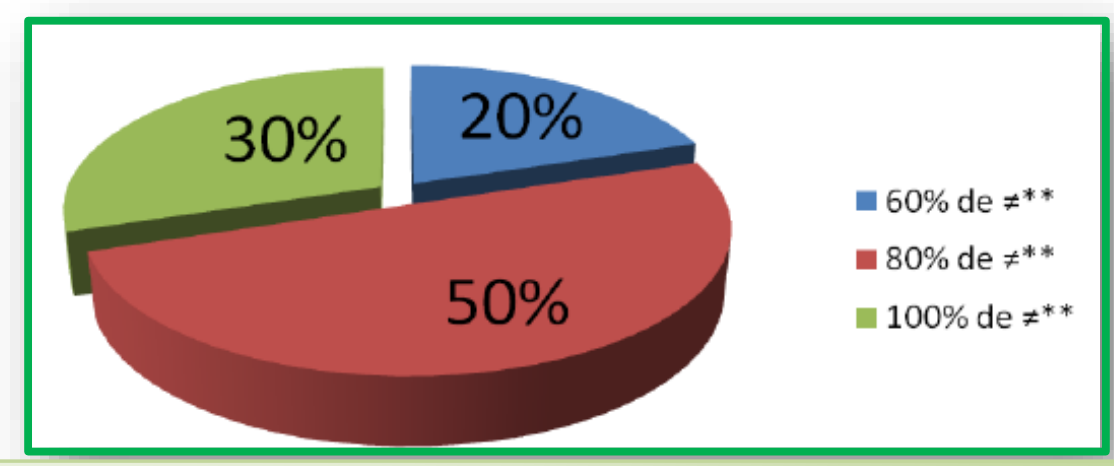

Figura 13. Distribución de la diferencia estadística en nitrógeno total entre las dos técnicas Diferencia $(\neq)$ Estadística $(P<0,0.5)$

En términos generales, en los resultados de las pruebas de digestibilidad in situ e in vitro se encontró diferencia $(P<0,005)$ entre los promedios obtenidos mediante estas dos técnicas, Además se encontró en el $63,333 \%$ de los casos una mejor tasa de degradación mediante la técnica de digestibilidad in situ, y esta diferencia se presentó debido a que la DER de la MS, FDN y NT (Tablas 11, 12 y 13) fue mayor en el $70 \%, 60 \%$ y $60 \%$ de los casos respectivamente, comparado con la in vitro. Los mayores valores encontrados mediante la técnica in situ podrían deberse a que esta técnica aseguraría una mezcla constante de las fases sólida y líquida de la digesta, dando lugar a que el forraje contenido en las bolsas se encuentre expuesto al ataque continuo de bacterias celulolíticas, resultando en una mayor degradación de las paredes celulares, lo cual llevaría a una estimación de digestibilidad mayor en comparación con la técnica in vitro, la cual se ve muy limitada por la superficie de contacto entre el líquido ruminal y el forraje, factor incide negativamente en la acción de los microorganismos ruminales. 
También hay que tener en cuenta que el alimento ingerido desaparece del tracto digestivo por dos rutas: digestión y pasaje. En consecuencia, estos dos procesos compiten por el mismo sustrato, de tal manera que existe la probabilidad de que una parte del material potencialmente digestible escape de la digestión y pase a las heces. En el caso de la incubación de sustratos en bolsas de nylon en el rumen de un animal, una parte del material fino, el más soluble en el fluido, escapará del rumen sin digerirse. Pero el material en frascos de incubación in vitro, no puede escapar y, en consecuencia, estará expuesto a la acción microbiana durante todo el período de incubación. De lo anterior se deduce que la desaparición de sustrato de las bolsas en el rumen debería ser mayor que la desaparición de sustrato en los frascos (Arreaza et al., 2005).

Los resultados de esta investigación difieren a los resultados reportados por Giraldo et al. (2007), en dicho estudio, los estimados de digestibilidad in vitro verdadera de la materia seca se compararon en cuatro forrajes de origen tropical usando el incubador ANKOM Daisy II $\circledast$ y la técnica in situ. Aunque en este estudio también se encontró diferencia significativa $(\mathrm{P}<0.05)$ en la digestibilidad verdadera in vitro de la materia seca (DVIVMS) entre los forrajes angleton, kikuyo y san Joaquín, la correlación entre la DVIVMS y la degradabilidad verdadera in situ de la materia seca (DVISMS), fue significativa y alta $\left(P<0.01, R^{2}=0.95\right)$ para los cuatro forrajes evaluados, y además, fue posible predecir la degradabilidad verdadera in situ de la materia seca con base en la digestibilidad verdadera in vitro de la materia seca mediante una ecuación. Finalmente se concluye que los resultados obtenidos, permiten confirmar que la técnica in vitro para estimar la digestibilidad verdadera utilizando el incubador Daisy II es confiable, rápida, precisa y sencilla, en comparación con el método in vivo utilizando la degradabilidad ruminal in situ.

Por otro lado, hay reportes de diferencia no solo entre las técnicas de digestibilidad in vitro e in situ, sino que también se reportan diferencias con otras técnicas de digestibilidad. De manera similar a los resultados de esta investigación, Torres et al. (2009), compararon las técnicas de digestibilidad in situ, in vitro y enzimática (celulasa) para estimar la digestibilidad de forrajes en ovinos, concluye que existen diferencias entre las técnicas in situ, in vitro y celulasa para estimar la digestibilidad de la MS del forraje en estos animales y que dichas diferencias dependen de la calidad del forraje. Así mismo, Arreaza et al. (2005) determinaron la degradabilidad ruminal de fracciones de carbohidratos en forrajes tropicales por métodos 
in situ e in vitro, reportan que las diferencias entre las degradaciones in situ e in vitro implican que hay errores en los procedimientos, o que estos dos métodos no son comparables, a pesar de existir una alta correlación entre ambos, y que las diferencias dentro de fracciones se acentúan sobre las menos solubles como la fibra detergente neutro. En este estudio se encontró diferencias altamente significativas entre los dos métodos y entre todas las fracciones. Así mismo, Arce et al., (2003) en un estudio comparativo de la digestibilidad de forrajes mediante dos métodos de laboratorio realizando la prueba T-Student pareada, reportan que los resultados de digestibilidad obtenidos por los métodos enzimático e in vitro fueron estadísticamente diferentes ( $P>0.001$ ). Se atribuyeron los hallazgos, por un lado, a la riqueza del inóculo ruminal, donde actúan todo un conjunto de enzimas provenientes de los diferentes microorganismos (bacterias, protozoarios y hongos) que causan una mayor degradación de los forrajes, mientras que el método enzimático trabaja solo con celulasa proveniente de un hongo, pero finalmente concluyen que existe una alta correlación entre el método enzimático y el método in vitro por lo que se recomienda el uso del método enzimático como una alternativa en el laboratorio para la evaluación de la digestibilidad de los forrajes.

\section{CONCLUSIONES}

En el Piedemonte llanero del Meta, existen diferencias entre las técnicas de digestibilidad in situ en bovinos rumino-fistulados, e in vitro para estimar la digestibilidad de la MS, FDN y NT del pasto amargo ( $B$. decumbens), kingrass ( $P$. purpureum), botón de oro ( $T$. diversifolia), casco de vaca (B. variegata), yopo Piptadenia peregrina), veranera (C. argentea), palo de cruz (B. ariza), cayeno (H. rosa-sinensis), matarratón (G. sepium) y acacia roja (D. regia). Hay diferencial total (100\%) entre las técnicas de digestibilidad in situ e in vito en los forrajes C. argentea, $B$. ariza y $G$. sepium; para los forrajes $H$. rosa-sinensis y $B$. variegata la diferencia fue del $86,667 \%$. De manera similar, en los forrajes $B$. decumbens y Piptadenia peregrina la diferencia fue del $80 \%$. Así mismo, en los forrajes $P$. purpureum, $T$. diversifolia y D. regia la diferencia fue del $73,333 \%$.

La diferencia entre los promedios de digestibilidad in situ e in vitro, de la MS fue del $100 \%$ en B. variegata, C. argentea, B. ariza, $H$. rosa-sinensis y $G$. sepium total; del $60 \%$ en $B$. decumbens, $P$. purpureum, $T$. diversifolia y Piptadenia peregrina; y en $D$. regia fue del $80 \%$. 
De igual manera, en la FDN fue del $100 \%$ en $B$. decumbens, $T$. diversifolia, $C$. argéntea, Piptadenia peregrina, B. ariza, $H$. rosa-sinensis y $G$. sepium; en $P$. purpureum y $B$. variegata fue del $80 \%$; y en $D$. regia fue del $60 \%$. Así mismo, para el caso del NT fue del $100 \%$ en $C$. argéntea, B. ariza y $G$. sepium; en $P$. purpureum, B. variegata, Piptadenia peregrina y $D$. regia fue del $80 \%$; y en $T$. diversifolia e $H$. rosa-sinensis fue del $60 \%$.

En el $63,333 \%$ de los casos se encontró una mejor tasa de degradación mediante la técnica in situ, y esta diferencia se presentó debido a que la DER de la MS, FDN y NT fue mayor en el $70 \%, 60 \%$ y $60 \%$ de los casos respectivamente. Por otro lado, el nitrógeno amoniacal fue más bajo en el líquido ruminal del animal de mejor condición corporal, en donde se incubaron las especies forrajeras de mayor contenido proteico $T$. diversifolia, C. argentea, G. sepium, $H$. rosa-sinensis y $D$. regia, excepto a las 6 horas de incubación. El pH fue más alto en el líquido de este mismo animal, excepto a las 12 horas de incubación.

\section{BIBLIOGRAFÍA}

1. Association of Official Analytical Chemists (A.O.A.C.). Official Methods of Analysis. 18th Ed., Washington, D. C. 2006.

2. Arce C, Arbaiza T, Carcelen F, Lucas O. Estudio comparativo de la digestibilidad en forrajes mediante dos métodos de laboratorio. Rev de Investigaciones Veterinarias Perú 2003: 14 (1): 7-12.

3. Arreaza LC, Sánchez DE, Abadía B. Degradabilidad ruminal de fracciones de carbohidratos en forrajes tropicales determinada por métodos in vitro e in situ. Rev CORPOICA. 2005 (6): 52-57.

4. Bochi-Brum O, Carro MD, Valdés C, González JS, López S. Digestibilidad in vitro de forrajes y concentrados: efecto de la ración de los animales donantes de líquido ruminal. Arch Zoot. 1999: 48: 51-61.

5. Fernández $\mathrm{HH}$. Un procedimiento simple para estimar parámetros de funciones útiles en nutrición animal usando solver de excel. Rev Argentina de Prod Anim 2004: 24(12): 75-81.

6. Giraldo C, Valderrama E, Montoya L, Armbrecht I. Efecto de Tithonia diversifolia (Asteraceae) sobre herbivoría de Atta cephalotes (Hymenoptera: Formicidae). En: Resúmenes IV Congreso Latinoamericano de Agroforestería para la producción animal sostenible y III Simposio sobre sistemas silvopastoriles para la producción ganadera sostenible. EEPF “Indio Hatuey". Matanzas, Cuba. Enero, 2006:113.

7. Giraldo LA, Gutiérrez LA, Rúa C. Comparación de dos técnicas: in vitro e in situ para estimar la digestibilidad verdadera en varios forrajes tropicales. Rev Colom de Cien Pecu. 2007: 20:269-279.

8. Mertens DR. Gravimetric Determination of Amylase-treated Neutral Detergent Fiber in Feeds with Refluxing in beakers or Crucibles: Collaborative Study. J of AOAC International. 2002: 85 (6): 1217-1240.

9. Rosero R, Posada, S. Modelación de la cinética de degradación de alimentos para rumiantes. Rev Colom de Cien Pecu. 2007: 20: (2): 174-182.

10. Roa ML, Castillo CA, Téllez E. Influencia del tiempo de maduración en la calidad nutricional, de ensilajes con forrajes arbóreos. Sist de Prod Agroec. 2010: 1 (1): 63-73.

11. Sosa D, Larco C, Falconi R, Toledo D, Suarez G. Digestibilidad de maralfalfa (Pennisetum sp.) en cabras. IASA. Boletín Técnico, Serie Zoológica. 2006: 5 (2): 68-76.

12. Tilley JM, Terry RA. A two-stage technique for the in vitro digestion of forage crops. En: This Week's Citation Classic. 1980: 15: $1 p$

13. Torres G, Arbaiza T, Carcelen F, Lucas F. Comparación de las técnicas in situ, in vitro y enzimática (Celulasa) para estimar la digestibilidad de forrajes en ovinos. Rev Inv Vet Perú. 2009:20 (1) 5-9.

14. Van Soest PJ, Robertson JB, Lewis BA. Symposium: carbohydrate methodology, metabolism, and nutritional implications in dairy cattle methods for dietary fiber, neutral detergent fiber, and nonstarch polysaccharides in relation to animal nutrition. J dairy sci. 1991:74:3583-3597. 